\title{
A DWINDLING FILTER LINE SEARCH METHOD FOR UNCONSTRAINED OPTIMIZATION
}

\author{
YANNAN CHEN AND WENYU SUN
}

\begin{abstract}
A dwindling multidimensional filter is proposed and applied to a second-order line search framework for unconstrained optimization. Usually, the multidimensional filter is built up with a fixed envelope, which is not well-suited to line search frameworks. In this paper, we propose the dwindling multidimensional filter, whose envelope is dwindling as the step-length of line search decreasing. Combining the dwindling multidimensional filter and a second-order line search, the new algorithm globally converges to a second-order critical point, when the negative curvature direction is exploited. Detailed numerical results on small and large CUTE test problems indicate that the new algorithm is more competitive than some classical line search methods.
\end{abstract}

\section{INTRODUCTION}

We consider the unconstrained optimization problem

$$
\min _{x \in \mathbb{R}^{n}} f(x),
$$

where $f$ is a real-valued function on $\mathbb{R}^{n}$. We assume that both the gradient $g(x):=$ $\nabla_{x} f(x)$ and the Hessian $H(x):=\nabla_{x x}^{2} f(x)$ of $f$ exist and are continuous. In this paper, we use the following notations: $\|\cdot\|$ is the Euclidean norm, $|\cdot|$ is the absolute value, $\left\{x_{k}\right\}$ a sequence of points generated by an algorithm, $f_{k}:=f\left(x_{k}\right)$, $g_{k}:=g\left(x_{k}\right), H_{k}:=H\left(x_{k}\right)$, and so on.

The filter method was first introduced for constrained nonlinear programming by Fletcher and Leyffer [9], which is a method without any penalty function and guarantees global convergence $[8,10,12,18,29,30,34,35]$. For unconstrained optimization, there are mainly two types of filter methods. The first approach is a straightforward application of the filter technique; see [21, 26, 36]. In particular, Wang and Zhu [36] transform the simple unconstrained optimization problem (1.1) to a nonlinear programming problem with an equality constraint

$$
\min _{x \in \mathbb{R}^{n}} f(x) \quad \text { s.t. } \quad g(x)=0,
$$

Received by the editor January 12, 2011 and, in revised form, December 25, 2012 and May 1, 2013.

2010 Mathematics Subject Classification. Primary 65K05, 90C30.

Key words and phrases. Filter method, line search, negative curvature direction, unconstrained optimization, global convergence.

This work was supported by the National Natural Science Foundation of China (Nos. 11171159 and 11071122), the Specialized Research Fund for the Doctoral Program of Higher Education of China (No. 20103207110002), and the Graduate Student Research and Innovation Project of Jiangsu Province of China (No. CXZZ12_0384). 
and then the filter technique for general nonlinear programming is applied. The second approach is the multidimensional filter technique, which is proposed for nonlinear equations and nonlinear least-squares in [13] and for unconstrained optimization in [16. The multidimensional filter has more freedom in accepting trial points, and has been extensively studied in [17, 19, 24, 37, 38. A brief history and review of filter methods are given in [11,31.

A multidimensional filter $\mathcal{F}$ is defined as a list of $n$-tuples $\left(g_{k, 1}, \ldots, g_{k, n}\right)$, where $g_{k, i}:=g_{i}\left(x_{k}\right)$, such that if $g_{k}$ and $g_{l}$ belong to $\mathcal{F}$, then $\left|g_{k, j}\right|<\left|g_{l, j}\right|$ holds for at least one $j \in\{1, \ldots, n\}$. A trial point $x_{k}^{+}$, whose gradient is denoted as $g_{k}^{+}:=$ $\left(g_{k, 1}^{+}, \ldots, g_{k, n}^{+}\right)$, is acceptable for the multidimensional filter $\mathcal{F}$ if and only if

$$
\begin{array}{r}
\text { for all } g_{l} \in \mathcal{F}, \text { there exists a } j \in\{1, \ldots, n\} \\
\text { such that }\left|g_{k, j}^{+}\right|-\left|g_{l, j}\right| \leq-\gamma \| g_{l}||,
\end{array}
$$

where $\gamma \in(0,1 / \sqrt{n})$ is a small positive constant. We say that $\gamma\left\|g_{l}\right\|$ is a fixed measurement of the envelope of the multidimensional filter $\mathcal{F}$.

Next, many line search algorithms 32 aim to attain a second-order critical point $x^{*}$ satisfying $g\left(x^{*}\right)=0$, and $H\left(x^{*}\right)$ is positive semidefinite. The key idea of these second-order line search algorithms is to determine, at each iterate $x_{k}$, a pair of descent directions $\left(s_{k}, d_{k}\right)$. Here, $s_{k}$ is a gradient-related direction satisfying

$$
s_{k}^{\top} g_{k} \leq-c_{1}\left\|g_{k}\right\|^{2} \quad \text { and } \quad\left\|s_{k}\right\| \leq c_{2}\left\|g_{k}\right\|,
$$

where $c_{1}$ and $c_{2}$ are two positive constants. The negative curvature direction $d_{k}$ satisfies

$$
\begin{aligned}
& d_{k}^{\top} g_{k} \leq 0, \quad d_{k}^{\top} H_{k} d_{k} \leq 0, \\
& d_{k}^{\top} H_{k} d_{k} \rightarrow 0 \quad \text { implies } \quad \min \left(0, \lambda_{\min }\left(H_{k}\right)\right) \rightarrow 0,
\end{aligned}
$$

where $\lambda_{\min }(H)$ is the smallest eigenvalue of the matrix $H$. Traditionally, secondorder line search algorithms use these two directions simultaneously, and process a curvilinear line search [23,25],

$$
x_{k+1}=x_{k}+\alpha_{k}^{2} s_{k}+\alpha_{k} d_{k},
$$

where $\alpha_{k}>0$ is a step-length. Combining the second-order line search method and a nonmonotone technique, several efficient algorithms have been proposed for small and large scale optimization problems [2, 7, 22, 33, 39, 40, The scaling of two directions $s_{k}$ and $d_{k}$ is taken into account in [14, which selects the more promising one of these two directions instead of using two directions synchronously.

In our point of view, the fixed envelope of a multidimensional filter keeps an acceptable trial point far from iterates in the filter, which works well in a trust region framework. However, a tiny step-length may take place in a line search iteration. Then, suppose the current iterate has been added into the multidimensional filter, the trial point with a tiny step-length must get close to the current iterate and be rejected by the multidimensional filter due to its fixed envelope. So we think that the fixed envelope of a multidimensional filter is not suitable well to the line search process.

We propose a dwindling multidimensional filter which does not require the envelope involved being fixed. Under the dwindling filter's rule and some mild conditions, the trial point with a sufficiently small step-length cannot be rejected by the current iterate if it was added to the filter. So the dwindling filter proposed here is meaningful and important. Therefore, we apply the dwindling filter to a 
second-order line search algorithm, and give a new algorithm to solve unconstrained optimization.

This paper is organized as follows. We present the dwindling multidimensional filter and its motivation in Section 2. The dwindling filter line search algorithm is proposed in Section 3. The corresponding convergence analysis to a second-order critical point is proved in Section 4. In Section 5, we report some preliminary numerical experiments on a collection of CUTE test problems. Some conclusions are finally described in Section 6.

\section{The DWIndLing FILTER}

Before giving the definition of the dwindling multidimensional filter, we compare the rule of the original multidimensional filter with a descent condition for the inexact line search, and introduce our motivation.

2.1. Motivation. First, we examine the inexact line search method. Suppose that the current iterate is $x_{k}$, the line search direction is a gradient-related direction $s_{k}$ that satisfies (1.3), and the trial point is

$$
x_{k}^{+}(\alpha):=x_{k}+\alpha s_{k} .
$$

We denote its objective function as $f_{k}^{+}(\alpha):=f\left(x_{k}^{+}(\alpha)\right)$ and its gradient as $g_{k}^{+}(\alpha):=$ $g\left(x_{k}^{+}(\alpha)\right)$ with components $g_{k, j}^{+}(\alpha)$ for $j=1, \ldots, n$. The trial point $x_{k}^{+}(\alpha)$ is acceptable for the inexact line search only if

$$
f_{k}^{+}(\alpha)-f_{k} \leq \mu \cdot \alpha g_{k}^{\top} s_{k}, \quad \mu \in\left(0, \frac{1}{2}\right)
$$

Note that, from the condition (1.3) and the Cauchy-Schwarz inequality, we have

$$
-c_{2}\left\|g_{k}\right\|^{2} \leq g_{k}^{\top} s_{k} \leq-c_{1}\left\|g_{k}\right\|^{2} .
$$

Hence, the line search rule (2.1) has two properties.

(i) The least decrease of the objective function $\mu \alpha g_{k}^{\top} s_{k}$ is proportional to the step-length $\alpha$ and $\mathcal{O}\left(\left\|g_{k}\right\|^{2}\right)$.

(ii) Inequality (2.1) holds when $\alpha$ is sufficiently small; see [28, 32.

Second, we consider a simple example which shows that the multidimensional filter (1.2) is not well-suited in a line search framework.

Example 2.1. Suppose that $f(x)$ is a one-variable function, i.e., $n=1$, and the current iterate $x_{k}$, whose gradient $g_{k} \neq 0$, belongs to the original multidimensional filter. By the rule (1.2), the trial point $x_{k}^{+}(\alpha)$ is acceptable if and only if

$$
\left|g_{k}^{+}(\alpha)\right|-\left|g_{k}\right| \leq-\gamma\left|g_{k}\right| \text {. }
$$

Then, we find that

(i) The least decrease $\gamma\left|g_{k}\right|$ in (2.2) is irrelevant to the line search step-length $\alpha$.

(ii) The inequality (2.2) does not hold if the step-length $\alpha$ is tiny.

This fact means that according to the rule of multidimensional filter (1.2), the trial point $x_{k}^{+}(\alpha)$ must be rejected if the current iterate $x_{k}$ is included in the filter $\mathcal{F}$ and the step-length $\alpha$ is sufficiently small. 
In our viewpoint, compared with the rule (2.1) of the inexact line search, the multidimensional filter's rule (1.2) is crude for line search methods. Then, we give a modification and improvement, which is the dwindling multidimensional filter.

2.2. The dwindling multidimensional filter. Our idea is to introduce the steplength factor into the rule of multidimensional filter and make the envelope of the filter become thinner and thinner as the step-length approaches zero. For a twovariable problem, we illustrate the idea in Figure 1.

Now, we formally present the new rule for the dwindling multidimensional filter. A trial point $x_{k}^{+}(\alpha)$ is acceptable to the dwindling filter $\mathcal{F}$ if and only if it satisfies

$$
\begin{array}{r}
\text { for all } g_{l} \in \mathcal{F}, \text { there exists a } j \in\{1, \ldots, n\} \\
\text { such that }\left|g_{k, j}^{+}(\alpha)\right|-\left|g_{l, j}\right| \leq-\gamma \phi(\alpha)\left\|g_{l}\right\|,
\end{array}
$$

where $\gamma \in\left(0, \frac{1}{\sqrt{n}}\right]$ is a constant and $\phi(\alpha)$ is a dwindling function defined as follows.

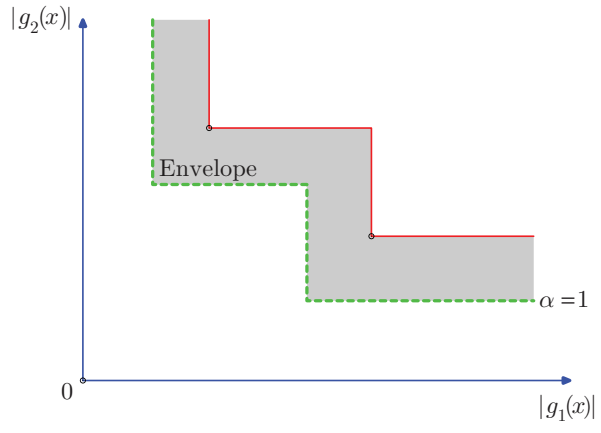

(a)

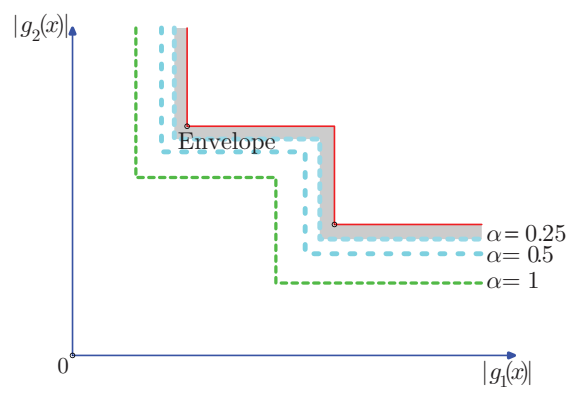

(c)

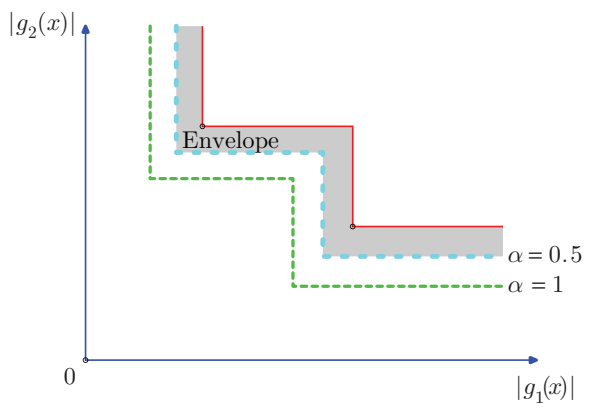

(b)

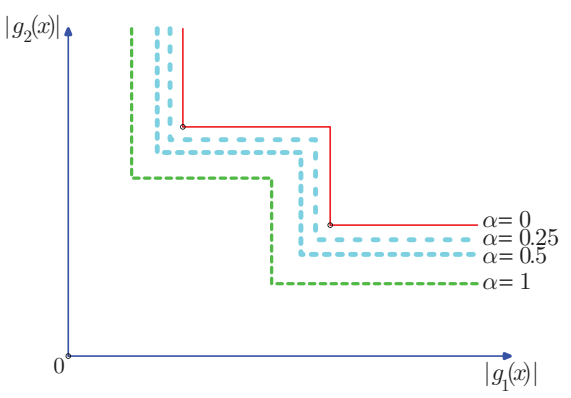

(d)

FiguRE 1. The envelopes of the dwindling multidimensional filter vary as a line search step-length $\alpha$ decreases. (a) When $\alpha$ is the unit step-length, the envelope is substantial and is equal to the original multidimensional filter's envelope. The envelopes become thinner as $\alpha=0.5$ in (b) and $\alpha=0.25$ in (c). (d) If $\alpha$ vanishes, the envelope vanishes, too. 
Definition 2.2. A continuous function $\phi(\alpha):[0,1] \mapsto \mathbb{R}^{+}$is a dwindling function if it is monotone increasing and satisfies $\phi(\alpha)=0$ if and only if $\alpha=0$. Moreover, we suppose that

$$
\lim _{\alpha \rightarrow 0^{+}} \frac{\phi(\alpha)}{\alpha}=0 .
$$

Obviously, if $\phi(\alpha) \equiv 1$, the dwindling filter reduces to the original multidimensional filter.

For large-scale problems, checking the dwindling filter's rule (2.3) in each component is a time-consuming work. Next, we give a sufficient condition for (2.3). This lemma also explains why we set $\gamma \in\left(0, \frac{1}{\sqrt{n}}\right]$ in a multidimensional filter.

Lemma 2.3. Suppose that the trial point $x_{k}^{+}(\alpha)$ satisfies

$$
\frac{\left\|g_{k}^{+}(\alpha)\right\|}{\left\|g_{l}\right\|} \leq 1-\sqrt{n} \gamma \phi(\alpha)
$$

where $x_{l}$ is a point included in the dwindling filter. Then, by the dwindling filter's rule (2.3), $x_{k}^{+}(\alpha)$ could not be rejected by $x_{l}$.

Proof. For contradiction, we suppose that $x_{k}^{+}(\alpha)$ is rejected by $x_{l}$, then

$$
\left|g_{k, j}^{+}(\alpha)\right|-\left|g_{l, j}\right|>-\gamma \phi(\alpha)\left\|g_{l}\right\|, \quad \text { for all } j=1, \ldots, n \text {. }
$$

Hence, we have

$$
\begin{aligned}
\left\|g_{k}^{+}(\alpha)\right\|-\left\|g_{l}\right\| & =\frac{\left\|g_{k}^{+}(\alpha)\right\|^{2}-\left\|g_{l}\right\|^{2}}{\left\|g_{k}^{+}(\alpha)\right\|+\left\|g_{l}\right\|} \\
& =\frac{\sum_{j=1}^{n}\left(\left|g_{k, j}^{+}(\alpha)\right|-\left|g_{l, j}\right|\right)\left(\left|g_{k, j}^{+}(\alpha)\right|+\left|g_{l, j}\right|\right)}{\left\|g_{k}^{+}(\alpha)\right\|+\left\|g_{l}\right\|} \\
& >\frac{-\gamma \phi(\alpha)\left\|g_{l}\right\| \sum_{j=1}^{n}\left(\left|g_{k, j}^{+}(\alpha)\right|+\left|g_{l, j}\right|\right)}{\left\|g_{k}^{+}(\alpha)\right\|+\left\|g_{l}\right\|} \\
& =\frac{-\gamma \phi(\alpha)\left\|g_{l}\right\|\left(\left\|g_{k}^{+}(\alpha)\right\|_{1}+\left\|g_{l}\right\|_{1}\right)}{\left\|g_{k}^{+}(\alpha)\right\|+\left\|g_{l}\right\|} \\
& \geq-\sqrt{n} \gamma \phi(\alpha)\left\|g_{l}\right\|,
\end{aligned}
$$

where $\|g\|_{1}$ is the 1 -norm satisfying $\|g\|_{1} \leq \sqrt{n}\|g\|$. This inequality contradicts (2.5). Hence, this lemma is proved.

It is easy to see that the condition (2.5) in Lemma 2.3 is strong. Therefore, we present a mild condition under which a trial point $x_{k}^{+}(\alpha)$ cannot be rejected by the current iterate $x_{k}$ when the dwindling filter is employed.

Lemma 2.4. Suppose that there exist an index $j \in\{1, \ldots, n\}$ and a small positive upper bound $\bar{\alpha}$ such that

$$
\left.g_{k, j}^{+}(\alpha) \cdot g_{k, j}>0, \quad \mid g_{k, j}^{+}(\alpha)\right)|<| g_{k, j} \mid \quad \text { and } \quad\left|e_{j}^{\top} H_{k} s_{k}\right| \neq 0
$$

hold for all $\alpha \in(0, \bar{\alpha}]$, where $e_{j}$ is the $j$-th column of the identity matrix, and the dwindling function $\phi(\alpha)$ satisfies (2.4). Then, under the dwindling filter's rule (2.3), the trial point $x_{k}^{+}(\alpha)$ could not be rejected by the current iterate $x_{k}$ if the step-length $\alpha$ is sufficiently small. 
Proof. Since $\left|g_{k, j}\right|>0$, we have $\left\|g_{k}\right\| \neq 0$. Then, from the assumptions and Taylor's theorem, we could obtain

$$
\begin{aligned}
\left|g_{k, j}^{+}(\alpha)\right|-\left|g_{k, j}\right| & =-\left|g_{k, j}^{+}(\alpha)-g_{k, j}\right| \\
& =-\left|e_{j}^{\top}\left(g_{k}^{+}(\alpha)-g_{k}\right)\right| \\
& =-\left|\alpha e_{j}^{\top} H_{k} s_{k}+o(\alpha)\right| \\
& =-\alpha\left|e_{j}^{\top} H_{k} s_{k}\right|+o(\alpha) .
\end{aligned}
$$

Since $\left|e_{j}^{\top} H_{k} s_{k}\right| \neq 0$ and $\bar{\alpha}$ is small, without loss of generality, we suppose

$$
\left|g_{k, j}^{+}(\alpha)\right|-\left|g_{k, j}\right| \leq-\frac{1}{2} \alpha\left|e_{j}^{\top} H_{k} s_{k}\right|
$$

holds for all $\alpha \in(0, \bar{\alpha}]$. By the definition (2.4) of $\phi(\cdot)$, we have

$$
\lim _{\alpha \rightarrow 0^{+}} \frac{-\gamma \phi(\alpha)\left\|g_{k}\right\|}{\left|g_{k, j}^{+}(\alpha)\right|-\left|g_{k, j}\right|}=\lim _{\alpha \rightarrow 0^{+}} \frac{\gamma \phi(\alpha)\left\|g_{k}\right\|}{\left|g_{k, j}\right|-\left|g_{k, j}^{+}(\alpha)\right|} \leq \lim _{\alpha \rightarrow 0^{+}} \frac{\phi(\alpha) \gamma\left\|g_{k}\right\|}{\alpha\left|e_{j}^{\top} H_{k} s_{k}\right| / 2}=0<1 .
$$

Therefore, once the step-length $\alpha$ is small enough, the dwindling filter's rule (2.3) holds and $x_{k}^{+}(\alpha)$ cannot be rejected by $x_{k}$.

\section{THE DWINDLING FILTER LINE SEARCH ALGORITHM}

Now, we combine the dwindling multidimensional filter with a second-order line search and give a new algorithm for unconstrained optimization.

The main improvement is on the switching condition, which selects one descent direction from a gradient-related direction and a negative curvature direction for the following line search process. We employ a common local merit function

$$
m_{k}(p):=g_{k}^{\top} p+\frac{1}{2} \min \left(p^{\top} H_{k} p, 0\right)
$$

and formulate the new switching condition as

$$
m_{k}\left(s_{k}\right) \leq \tau m_{k}\left(d_{k}\right),
$$

where $\tau$ is a positive constant. If the switching condition (3.2) holds, the local quadratic model is approximately convex. So we select the gradient-related direction. Otherwise, we perform a line search along a negative curvature direction.

It is worthwhile to note that these two directions used in the switching condition (3.2) are not normalized to the same unit norm. In this way, the decrease of the local merit function (3.1) in each selected direction has a negative upper bound if the current iterate is not critical. That is to say, whether the selected direction $p_{k}=s_{k}$ or $p_{k}=d_{k}$, we have

$$
m_{k}\left(p_{k}\right) \leq-\min \left(\tau^{-1}, 1\right) c_{1}\left\|g_{k}\right\|^{2} .
$$

For the purpose of avoiding an abnormal norm of the negative curvature direction $d_{k}$, we scale $d_{k}$ by its Rayleigh quotient

$$
d_{k} \leftarrow \frac{\left|d_{k}^{\top} H_{k} d_{k}\right|}{\left\|d_{k}\right\|^{3}} d_{k}
$$

Obviously, the scaled $d_{k}$ also satisfies (1.4).

The dwindling filter line search algorithm is described in Algorithm 1 A trial point is acceptable by the dwindling filter only if the local quadratic model is convex. 


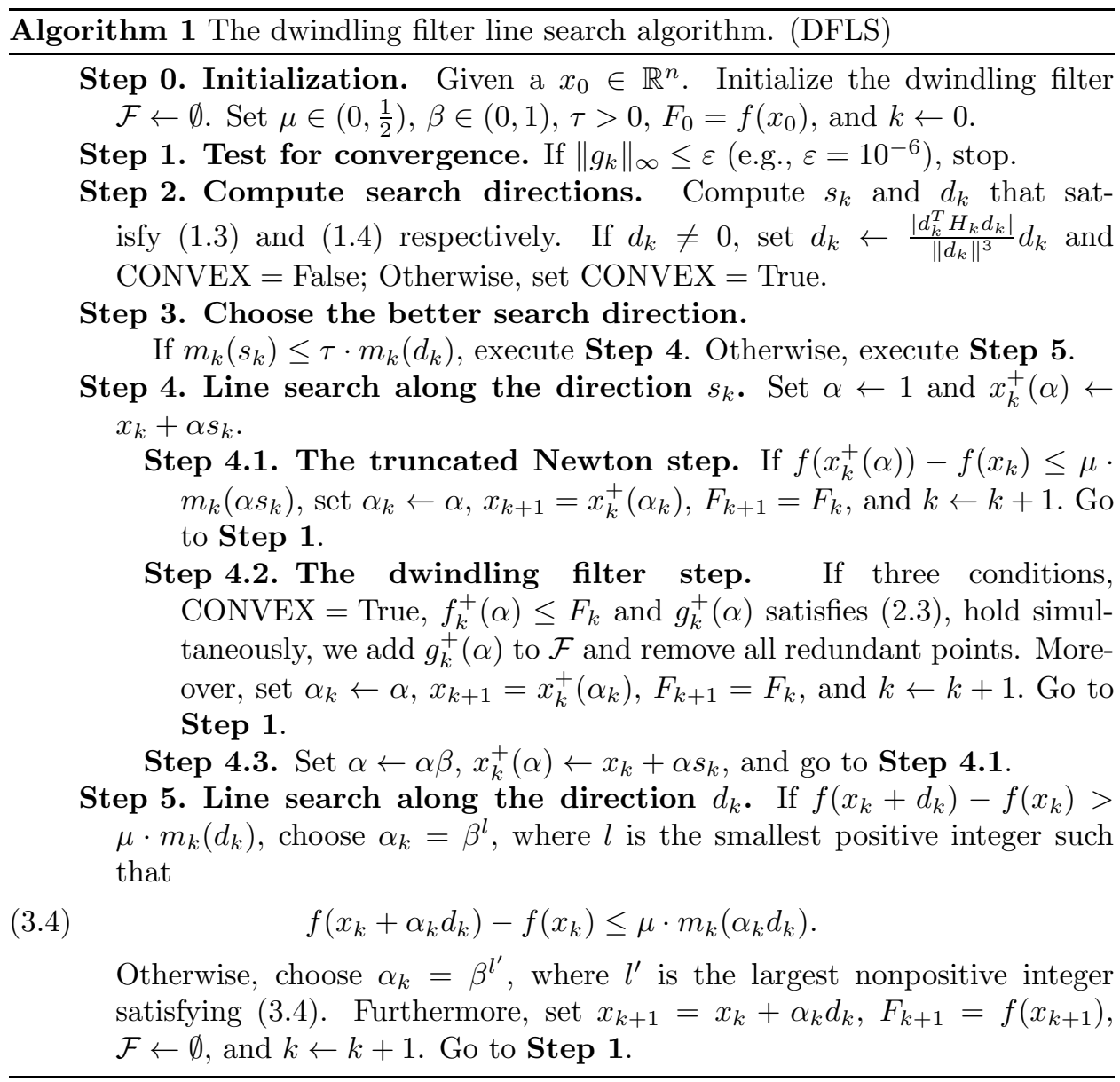

If the trial point $x_{k}^{+}\left(\alpha_{k}\right)$ is accepted by the dwindling filter, we add $g_{k}^{+}\left(\alpha_{k}\right)$ to the filter and remove from the filter each point $g_{l}$ satisfying

$$
\left|g_{l, j}\right|>\left|g_{k, j}^{+}\left(\alpha_{k}\right)\right| \text { for all } j \in\{1, \ldots, n\} .
$$

Additionally, when the current local quadratic model is seriously nonconvex, i.e., the switching condition (3.2) fails, we clear out the dwindling filter.

From Lemma 2.1 of reference [14, the line search process along either the gradient-related direction or the negative curvature direction is well defined. That is to say, there is no endless loop.

\section{Convergence analysis}

At the beginning of the analysis, we give a fundamental assumption.

Assumption 4.1. The objective function $f: \mathbb{R}^{n} \mapsto \mathbb{R}$ is twice continuously differentiable, and the level set $\Omega:=\left\{x \in \mathbb{R}^{n} \mid f(x) \leq f\left(x_{0}\right)\right\}$ is compact for the given initial point $x_{0}$.

Owing to Assumption 4.1, all the iterates $\left\{x_{k}\right\}_{k=0}^{\infty}$, objective functions $\left\{f_{k}\right\}_{k=0}^{\infty}$, gradients $\left\{g_{k}\right\}_{k=0}^{\infty}$, and Hessians $\left\{H_{k}\right\}_{k=0}^{\infty}$ generated by the DFLS algorithm are 
bounded. Additionally, by (1.3) and (3.3), directions $\left\{s_{k}\right\}_{k=0}^{\infty}$ and $\left\{d_{k}\right\}_{k=0}^{\infty}$ are bounded, too.

For the convenience of the following analysis, we formally set a (sufficiently large) positive constant $M$ such that

$$
\left\|H_{k}\right\| \leq M \quad \text { for all } k \text {. }
$$

We suppose that there are infinite iterations produced by the DFLS algorithm. According to the switching condition (3.2), either the gradient-related direction $s_{k}$ or the negative curvature direction $d_{k}$ is used in each iteration. First, we examine the case in which $d_{k}$ is employed, infinitely many times.

Theorem 4.2. Suppose Assumption 4.1 holds. Let

$$
\mathcal{K}_{d}:=\left\{k \mid m_{k}\left(s_{k}\right)>\tau m_{k}\left(d_{k}\right)\right\}
$$

be an infinite index set. Then, every limit point of $\left\{x_{k}\right\}_{k \in \mathcal{K}_{d}}$ is a critical point, i.e.,

$$
\lim _{k \rightarrow \infty, k \in \mathcal{K}_{d}}\left\|g_{k}\right\|=0 .
$$

Proof. Let $\mathcal{K}_{d}=\left\{k_{i}\right\}_{i=1}^{\infty}$. According to Step 5 of the DFLS algorithm, we have

$$
f\left(x_{k_{i}}\right)>f\left(x_{k_{i}}\right)+\mu m_{k_{i}}\left(\alpha_{k_{i}} d_{k_{i}}\right) \geq f\left(x_{k_{i}+1}\right)=F_{k_{i}+1}=\cdots=F_{k_{i+1}} \geq f\left(x_{k_{i+1}}\right) .
$$

Hence,

$$
f\left(x_{k_{i+1}}\right)-f\left(x_{k_{i}}\right) \leq \mu m_{k_{i}}\left(\alpha_{k_{i}} d_{k_{i}}\right) \leq \mu \cdot \min \left(\alpha_{k_{i}}, \alpha_{k_{i}}^{2}\right) m_{k_{i}}\left(d_{k_{i}}\right)<0 .
$$

Since $f(x), x \in \Omega$, is bounded below, the difference $f\left(x_{k_{i+1}}\right)-f\left(x_{k_{i}}\right)$ must tend to zero as $i \rightarrow \infty$. So either $\alpha_{k_{i}} \rightarrow 0$ or $m_{k_{i}}\left(d_{k_{i}}\right) \rightarrow 0$ as $i \rightarrow \infty$ and $k_{i} \in \mathcal{K}_{d}$.

Suppose that there is an index subset $\mathcal{K}_{d}^{0} \subseteq \mathcal{K}_{d}$ such that

$$
\lim _{k \rightarrow \infty, k \in \mathcal{K}_{d}^{0}} \alpha_{k}=0 .
$$

Without loss of generality, we assume $\alpha_{k} \leq \beta$ for all $k \in \mathcal{K}_{d}^{0}$. For convenience, we define $\widehat{\alpha}_{k}:=\frac{\alpha_{k}}{\beta}$. Owing to the line search's rule, when $k \in \mathcal{K}_{d}^{0}$, we have

$$
f\left(x_{k}+\widehat{\alpha}_{k} d_{k}\right)-f\left(x_{k}\right)>\mu m_{k}\left(\widehat{\alpha}_{k} d_{k}\right) .
$$

By the mean-value theorem, we get

$$
\widehat{\alpha}_{k} g_{k}^{\top} d_{k}+\frac{1}{2} \widehat{\alpha}_{k}^{2} d_{k}^{\top} \widetilde{H}_{k} d_{k}>\mu \widehat{\alpha}_{k} g_{k}^{\top} d_{k}+\frac{\mu}{2} \widehat{\alpha}_{k}^{2} d_{k}^{\top} H_{k} d_{k},
$$

where $\widetilde{H}_{k}=H\left(x_{k}+\theta \widehat{\alpha}_{k} d_{k}\right)$ with $\theta \in(0,1)$. On the one hand, inequality (4.5) could be rewritten as

$$
g_{k}^{\top} d_{k}>\frac{\widehat{\alpha}_{k}}{2(1-\mu)}\left(\mu d_{k}^{\top} H_{k} d_{k}-d_{k}^{\top} \widetilde{H}_{k} d_{k}\right) .
$$

Since $g_{k}^{\top} d_{k} \leq 0$, and $\widehat{\alpha}_{k} \rightarrow 0$ as $k \rightarrow \infty, k \in \mathcal{K}_{d}^{0}$, we obtain

$$
\lim _{k \rightarrow \infty, k \in \mathcal{K}_{d}^{0}} g_{k}^{\top} d_{k}=0 .
$$

On the other hand, inequality (4.5) could also be rewritten as

$$
\frac{d_{k}^{\top} \widetilde{H}_{k} d_{k}-\mu d_{k}^{\top} H_{k} d_{k}}{1-\mu}>-\frac{2}{\widehat{\alpha}_{k}} g_{k}^{\top} d_{k} \geq 0 .
$$


When $k \rightarrow \infty, k \in \mathcal{K}_{d}^{0}$, we have $\widehat{\alpha}_{k} \rightarrow 0$ and $\widetilde{H}_{k} \rightarrow H_{k}$. So we have

$$
\lim _{k \rightarrow \infty, k \in \mathcal{K}_{d}^{0}} \frac{d_{k}^{\top} \widetilde{H}_{k} d_{k}-\mu d_{k}^{\top} H_{k} d_{k}}{1-\mu}=d_{k}^{\top} H_{k} d_{k} \leq 0 .
$$

Hence,

$$
\lim _{k \rightarrow \infty, k \in \mathcal{K}_{d}^{0}} d_{k}^{\top} H_{k} d_{k}=0 .
$$

Combining (4.6) and (4.7), we have

$$
\lim _{k \rightarrow \infty, k \in \mathcal{K}_{d}^{0}} m_{k}\left(d_{k}\right)=0 .
$$

Further, recalling (4.4), we obtain

$$
\lim _{k \rightarrow \infty, k \in \mathcal{K}_{d}} m_{k}\left(d_{k}\right)=0 .
$$

When $k \in \mathcal{K}_{d}$, we have

$$
m_{k}\left(d_{k}\right)<\tau^{-1} m_{k}\left(s_{k}\right) \leq \tau^{-1} g_{k}^{\top} s_{k} \leq-\tau^{-1} c_{1}\left\|g_{k}\right\|^{2}<0 .
$$

Hence, $\left\|g_{k}\right\| \rightarrow 0$ as $k \rightarrow \infty$ and $k \in \mathcal{K}_{d}$. The proof is complete.

Second, if the gradient-related direction is employed, the corresponding steplength has a positive lower bound.

Lemma 4.3. Suppose Assumption 4.1 holds. As the gradient-related direction $s_{k}$ is employed, the corresponding step-length satisfies

$$
\alpha_{k} \geq \alpha_{s}^{\min }:=\frac{(1-\mu) c_{1}}{2 M c_{2}^{2}}, \quad \text { for all } k \notin \mathcal{K}_{d} .
$$

Proof. By the mean-value theorem, we have

$$
g(x+s)-g(x)=H(x+\theta x) s, \quad \theta \in(0,1) .
$$

By (4.1), we obtain

$$
\|g(x+s)-g(x)\| \leq\|H(x+\theta x)\|\|s\| \leq M\|s\| .
$$

The inequality (4.10) means that the gradient is Lipschitz continuous.

When $k \notin \mathcal{K}_{d}$, by (4.10), (4.1), (1.3), and the mean-value theorem, we get

$$
\begin{aligned}
\mid f & \left(x_{k}+\alpha s_{k}\right)-f\left(x_{k}\right)-m_{k}\left(\alpha s_{k}\right) \mid \\
& =\left|\int_{0}^{1} g\left(x_{k}+t \alpha s_{k}\right)^{\top}\left(\alpha s_{k}\right) \mathrm{d} t-\alpha g_{k}^{\top} s_{k}-\frac{1}{2} \alpha^{2} \min \left(s_{k}^{\top} H_{k} s_{k}, 0\right)\right| \\
& \leq\left|\alpha \int_{0}^{1}\left(g\left(x_{k}+t \alpha s_{k}\right)-g_{k}\right)^{\top} s_{k} \mathrm{~d} t\right|+\frac{1}{2} \alpha^{2}\left|s_{k}^{\top} H_{k} s_{k}\right| \\
& \leq \alpha \int_{0}^{1}\left\|g\left(x_{k}+t \alpha s_{k}\right)-g_{k}\right\|\left\|s_{k}\right\| \mathrm{d} t+\frac{1}{2} \alpha^{2}\left\|H_{k}\right\|\left\|s_{k}\right\|^{2} \\
& \leq \alpha \int_{0}^{1} t M \alpha\left\|s_{k}\right\|^{2} \mathrm{~d} t+\frac{1}{2} \alpha^{2} M\left\|s_{k}\right\|^{2} \\
& =\alpha^{2} M\left\|s_{k}\right\|^{2} \\
& \leq \alpha^{2} M c_{2}^{2}\left\|g_{k}\right\|^{2} .
\end{aligned}
$$


In addition, from (1.3), we have

$$
\left|m_{k}\left(\alpha s_{k}\right)\right|=\left|\alpha g_{k}^{\top} s_{k}+\frac{1}{2} \alpha^{2} \min \left(s_{k}^{\top} H_{k} s_{k}, 0\right)\right| \geq\left|\alpha g_{k}^{\top} s_{k}\right| \geq \alpha c_{1}\left\|g_{k}\right\|^{2} .
$$

Combining (4.11) and (4.12), we obtain

$$
\frac{\left|f\left(x_{k}+\alpha s_{k}\right)-f\left(x_{k}\right)-m_{k}\left(\alpha s_{k}\right)\right|}{\left|m_{k}\left(\alpha s_{k}\right)\right|} \leq \alpha M c_{1}^{-1} c_{2}^{2} .
$$

Hence, when $\alpha M c_{1}^{-1} c_{2}^{2} \leq(1-\mu)$, i.e., $\alpha \leq \frac{(1-\mu) c_{1}}{M c_{2}^{2}}$, the inequality $f\left(x_{k}^{+}(\alpha)\right)-$ $f\left(x_{k}\right) \leq \mu m_{k}\left(\alpha s_{k}\right)$ is certainly valid. Therefore, this lemma holds.

Now, we consider the other case that $\mathcal{K}_{d}$ is a finite index set. Then, there exists an index

$$
k_{d}^{\max }:=\max \left\{k \mid k \in \mathcal{K}_{d}\right\} .
$$

When $k>k_{d}^{\max }$, the gradient-related direction is definitely exploited. According to the DFLS algorithm, the dwindling filter will never be cleared out when $k>k_{d}^{\max }$. In the following theorem, we examine a case in which there are infinite dwindling filter steps.

Theorem 4.4. Suppose Assumption 4.1 holds and

$$
\mathcal{K}_{f}:=\left\{k \mid k>k_{d}^{\max }, g_{k}^{+}\left(\alpha_{k}\right) \text { is added to } \mathcal{F}\right\}
$$

is an infinite index set. Then, every limit point of the sequence $\left\{x_{k}\right\}_{k \in \mathcal{K}_{f}}$ is a critical point, i.e.,

$$
\lim _{k \rightarrow \infty, k \in \mathcal{K}_{f}}\left\|g_{k}\right\|=0 .
$$

Proof. Suppose that there is an infinite subset $\left\{k_{i}\right\}_{i=1}^{\infty} \subseteq \mathcal{K}_{f}$ such that $\left\{x_{k_{i}}\right\}_{i=1}^{\infty}$ converges. So gradients $\left\{g_{k_{i}}\right\}_{i=1}^{\infty}$ converge, too. Therefore, we have

$$
\lim _{i \rightarrow \infty} g_{k_{i+1}, j}-g_{k_{i}, j}=0, \quad \text { for all } j=1, \ldots, n .
$$

According to the dwindling filter's rule, if

$$
\text { for all } j \in\{1, \ldots, n\}, \quad\left|g_{k_{i+1}, j}\right|-\left|g_{k_{i}, j}\right|>-\gamma \phi\left(\alpha_{k_{i+1}-1}\right)\left\|g_{k_{i}}\right\|,
$$

then $x_{k_{i+1}}$ is not acceptable and cannot be added to the dwindling filter. So there must exist $j_{i} \in\{1, \ldots, n\}$ such that

$$
\left|g_{k_{i+1}, j_{i}}\right|-\left|g_{k_{i}, j_{i}}\right| \leq-\gamma \phi\left(\alpha_{k_{i+1}-1}\right)\left\|g_{k_{i}}\right\| \leq-\gamma \phi\left(\alpha_{s}^{\min }\right)\left\|g_{k_{i}}\right\|<0 .
$$

By (4.15), we have $\left\|g_{k_{i}}\right\| \rightarrow 0$ as $i \rightarrow \infty$. We complete the proof.

Next, we consider the case that the index set $\mathcal{K}_{f}$ is also finite. Then, when $k$ is large enough, all the iterations are truncated Newton steps, and the sequence of objective values decreases monotonously.

Theorem 4.5. Suppose Assumption 4.1 holds and the index

$$
k_{f}^{\max }:=\max \left\{k \mid k \in \mathcal{K}_{f}\right\}
$$

is a finite integer. Then, every limit point of the sequence $\left\{x_{k}\right\}_{k>k_{f}^{\max }}$ is a critical point, i.e.,

$$
\lim _{k \rightarrow \infty}\left\|g_{k}\right\|=0
$$


Proof. Owing to the DFLS algorithm, when $k>k_{f}^{\max }$, we have

$$
f_{k+1}-f_{k} \leq \mu m_{k}\left(\alpha_{k} s_{k}\right) \leq \mu \alpha_{k} g_{k}^{\top} s_{k} \leq-\mu \alpha_{s}^{\min } c_{1}\left\|g_{k}\right\|^{2}<0 .
$$

Since $f(x), x \in \Omega$ is bounded below, so the difference $f_{k+1}-f_{k} \rightarrow 0$ as $k \rightarrow \infty$. Therefore, $\left\|g_{k}\right\| \rightarrow 0$ as $k \rightarrow \infty$.

Obviously, taking Theorems 4.2, 4.4 and 4.5 into account, we claim that the DFLS algorithm could find at least one first-order critical point.

Theorem 4.6. Suppose Assumption 4.1 holds. Then

$$
\liminf _{k \rightarrow \infty}\left\|g_{k}\right\|=0 \text {. }
$$

Finally, we give the second-order convergence result for DFLS algorithm.

Theorem 4.7. Suppose Assumption 4.1 holds and there is a unique limit point of the sequence of iterates $\left\{x_{k}\right\}_{k=0}^{\infty}$. Then, the unique limit point is a second-order critical point.

Proof. From Theorem 4.6, we get

$$
\lim _{k \rightarrow \infty}\left\|g_{k}\right\|=0
$$

By (1.3) and (4.1), we have

$$
\left|m_{k}\left(s_{k}\right)\right| \leq\left|g_{k}^{\top} s_{k}\right|+\left|\min \left(s_{k}^{\top} H_{k} s_{k}, 0\right)\right| \leq\left\|g_{k}\right\|\left\|s_{k}\right\|+M\left\|s_{k}\right\|^{2} \leq\left(c_{2}+M c_{2}^{2}\right)\left\|g_{k}\right\|^{2} .
$$

So $m_{k}\left(s_{k}\right) \rightarrow 0$ as $k \rightarrow \infty$.

We prove this theorem by contradiction. Suppose that there is a positive constant $\epsilon$ such that

$$
\frac{d_{k}^{\top} H_{k} d_{k}}{\left\|d_{k}\right\|^{2}} \leq-\epsilon \quad \text { for all } k \text { large enough. }
$$

Then, by the scaling (3.3) of $d_{k}$, we have

$$
m_{k}\left(d_{k}\right) \leq \frac{1}{2} d_{k}^{\top} H_{k} d_{k}=\frac{1}{2} \frac{d_{k}^{\top} H_{k} d_{k}}{\left\|d_{k}\right\|^{2}}\left\|d_{k}\right\|^{2}=\frac{1}{2}\left(\frac{d_{k}^{\top} H_{k} d_{k}}{\left\|d_{k}\right\|^{2}}\right)^{3} \leq-\frac{\epsilon^{3}}{2} .
$$

Hence, when $k$ is sufficiently large, we have $m_{k}\left(d_{k}\right)<\tau^{-1} m_{k}\left(s_{k}\right)$, i.e., $k \in \mathcal{K}_{d}$.

However, when $k \in \mathcal{K}_{d}$, recalling the limit (4.8) in Theorem 4.2, we find that the assumption (4.17) could not hold. Hence,

$$
\lim _{k \rightarrow \infty} d_{k}^{\top} H_{k} d_{k}=\lim _{k \rightarrow \infty}\left(\frac{d_{k}^{\top} H_{k} d_{k}}{\left\|d_{k}\right\|^{2}}\right)^{3}=0 .
$$

Owing to (1.4), we get $\min \left(0, \lambda_{\min }\left(H_{k}\right)\right) \rightarrow 0$ as $k \rightarrow \infty$. Therefore, this theorem is valid.

\section{NumericAl EXPERIMENTS}

In order to implement the DFLS algorithm, we employ the planar conjugate gradient method [4 6] to compute simultaneously a gradient-related direction and a negative curvature direction that satisfy (1.3) and (1.4) respectively. The planar conjugate gradient method is a sort of Krylov subspace iterative method which does not require an explicit Hessian matrix. Instead, a subroutine, which computes the product of the Hessian and an arbitrary vector, is enough. So it is suitable for largescale problems. Compared with the Lanczos method, the planar conjugate gradient 
method does not need to store or reproduce any matrix of Lanczos orthogonal basis. If the Hessian is sufficiently positive definite, the planar conjugate gradient method reduces to the general conjugate gradient method. It is cheap and fast.

The planar conjugate gradient method is terminated if the step $s_{k}$ of the local quadratic model satisfies

$$
\left\|H_{k} s_{k}+g_{k}\right\| \leq \max \left(\min \left(0.1, \sqrt{\left\|g_{k}\right\|}\right)\left\|g_{k}\right\|, \sqrt{\epsilon_{\operatorname{mach}}}\right)
$$

or the number of inner conjugate gradient iterations exceeds $\min (n, 30)$, where $\epsilon_{\text {mach }}$ is the machine precision. The parameters involved in the DFLS algorithm are set as follows:

$$
\mu=10^{-4}, \quad \beta=0.5, \quad \tau=1, \quad \gamma=\min \left(0.001, \frac{1}{2 \sqrt{n}}\right), \quad \phi(\alpha)=\alpha^{10} .
$$

We stop the algorithm if

$$
\left\|g_{k}\right\|_{\infty} \leq 10^{-6} \quad \text { or } \quad k \geq 10^{5} .
$$

To show the validity of the DFLS algorithm, we compare it with the adaptive line search (ALS) algorithm [14] and the state-of-the-art Limited memory BFGS (LBFGS) algorithm [20, 27]. In our experiments, the code of DFLS is written in Fortran 95 language. In the same language, we rewrite the code of ALS, where the two descent directions are also computed by the planar conjugate gradient method. The LBFGS code is downloaded from http://users.eecs.northwestern.edu/ nocedal/software.html\\#lbfgs.

In the remainder of this section, We illustrate numerical results by performance profiles, which is introduced by Dolan and Moré [3. The key point of performance profiles is the relative $\sigma$-optimum, where $\sigma \geq 1$ is an interesting parameter. Without loss of generality, we take the number of function evaluations, for example. Suppose that there are $N$ test problems. For each problem $i \in\{1,2, \ldots, N\}$, Algorithms DFLS, ALS and LBFGS solve it with the number of function evaluations $P_{(i)}^{D}$, $P_{(i)}^{A}$ and $P_{(i)}^{L}$, respectively. We say that DFLS for the problem $i$ is the relative $\sigma$-optimum if

$$
\sigma \geq \frac{P_{(i)}^{D}}{\min \left(P_{(i)}^{D}, P_{(i)}^{A}, P_{(i)}^{L}\right)}:=r_{(i)}^{D} .
$$

Obviously, an algorithm is excellent for the problem $i$ once $r_{(i)}^{D}$ takes a small value. Otherwise, if $\sigma$ is large and cannot be reduced, the algorithm for problem $i$ is disappointing. We define an identity function to show whether DFLS for problem $i$ is the relative $\sigma$-optimum

$$
\pi_{\sigma}^{D}(i):= \begin{cases}1 & \text { if } r_{(i)}^{D} \leq \sigma, \\ 0 & \text { otherwise. }\end{cases}
$$

Then, the possibility of DFLS for all problems being the relative $\sigma$-optimum is approximately

$$
p^{D}(\sigma)=\frac{\sum_{i=1}^{N} \pi_{\sigma}^{D}(i)}{N} .
$$


TABLE 1. The set of small-scale CUTE problems and their dimensions.

\begin{tabular}{|r|lr||r|lr||r|lr|}
\hline No. & Problem & $\mathrm{n}$ & No. & Problem & $\mathrm{n}$ & No. & Problem & $\mathrm{n}$ \\
\hline \hline 1 & AKIVA & 2 & 28 & DQDRTIC & 100 & 55 & NONDIA & 100 \\
2 & ALLINITU & 4 & 29 & ENGVAL1 & 100 & 56 & NONDQUAR & 100 \\
3 & ARWHEAD & 100 & 30 & ENGVAL2 & 3 & 57 & OSBORNEA & 5 \\
4 & BARD & 3 & 31 & ERRINROS & 50 & 58 & OSBORNEB & 11 \\
5 & BDQRTIC & 100 & 32 & EXPFIT & 2 & 59 & PALMER5C & 6 \\
6 & BEALE & 2 & 33 & EXTROSNB & 100 & 60 & PENALTY1 & 100 \\
7 & BOX & 100 & 34 & FLETCBV2 & 100 & 61 & PENALTY2 & 200 \\
8 & BOX3 & 3 & 35 & FLETCHCR & 100 & 62 & PENALTY3 & 200 \\
9 & BRKMCC & 2 & 36 & FREUROTH & 100 & 63 & POWELLSG & 100 \\
10 & BROWNAL & 200 & 37 & GENROSE & 500 & 64 & POWER & 100 \\
11 & BROWNBS & 2 & 38 & GULF & 3 & 65 & ROSENBR & 2 \\
12 & BROWNDEN & 4 & 39 & HAIRY & 2 & 66 & S308 & 2 \\
13 & BRYBND & 100 & 40 & HATFLDD & 3 & 67 & SCHMVETT & 100 \\
14 & CHNROSNB & 50 & 41 & HATFLDE & 3 & 68 & SINEVAL & 2 \\
15 & CLIFF & 2 & 42 & HATFLDFL & 3 & 69 & SISSER & 2 \\
16 & COSINE & 100 & 43 & HELIX & 3 & 70 & SPARSINE & 100 \\
17 & CUBE & 2 & 44 & HILBERTA & 2 & 71 & SPARSQUR & 100 \\
18 & CURLY10 & 100 & 45 & HILBERTB & 10 & 72 & TOINTGOR & 50 \\
19 & CURLY20 & 100 & 46 & HIMMELBB & 2 & 73 & TOINTGSS & 100 \\
20 & CURLY30 & 100 & 47 & HIMMELBF & 4 & 74 & TOINTPSP & 50 \\
21 & DENSCHNA & 2 & 48 & HIMMELBG & 2 & 75 & TOINTQOR & 50 \\
22 & DENSCHNB & 2 & 49 & HUMPS & 2 & 76 & TQUARTIC & 100 \\
23 & DENSCHNC & 2 & 50 & KOWOSB & 4 & 77 & TRIDIA & 100 \\
24 & DENSCHND & 3 & 51 & LIARWHD & 100 & 78 & VAREIGVL & 50 \\
25 & DENSCHNE & 3 & 52 & MARATOSB & 2 & 79 & WATSON & 12 \\
26 & DENSCHNF & 2 & 53 & MEXHAT & 2 & 80 & YFITU & 3 \\
27 & DIXON3DQ & 100 & 54 & MOREBV & 100 & & & \\
\hline
\end{tabular}

TABLE 2. The set of large-scale CUTE problems and their dimensions.

\begin{tabular}{|r|lr||r|lr||r|lr|}
\hline No. & Problem & $\mathrm{n}$ & No. & Problem & $\mathrm{n}$ & No. & Problem & $\mathrm{n}$ \\
\hline \hline 1 & ARWHEAD & 5000 & 9 & ENGVAL1 & 5000 & 17 & POWER & 10000 \\
2 & BDQRTIC & 5000 & 10 & EXTROSNB & 1000 & 18 & SCHMVETT & 5000 \\
3 & BOX & 10000 & 11 & FLETCHCR & 1000 & 19 & SPARSQUR & 10000 \\
4 & BRYBND & 5000 & 12 & LIARWHD & 5000 & 20 & SROSENBR & 5000 \\
5 & COSINE & 10000 & 13 & MOREBV & 5000 & 21 & TOINTGSS & 5000 \\
6 & DQDRTIC & 5000 & 14 & NONDIA & 5000 & 22 & TQUARTIC & 5000 \\
7 & EDENSCH & 2000 & 15 & PENALTY1 & 1000 & 23 & TRIDIA & 5000 \\
8 & EG2 & 1000 & 16 & POWELLSG & 5000 & 24 & WOODS & 4000 \\
\hline
\end{tabular}

Obviously, $p^{D}(\sigma)$ increases monotonously. If $p^{D}(\sigma)$ gets close to one fast, DFLS is a good algorithm. In a similar way, we define $p^{A}(\sigma)$ and $p^{L}(\sigma)$ for Algorithms ALS and LBFGS, respectively.

Using the performance profiles, we report numerical results of Algorithms DFLS, ALS and LBFGS for small-scale and large-scale CUTE test problems [1, 15]. Table 1 lists eighty small-scale problems whose dimensions are less than or equal to five hundred. All the problems use default initial points provided by the CUTE collection and find the same critical points. 


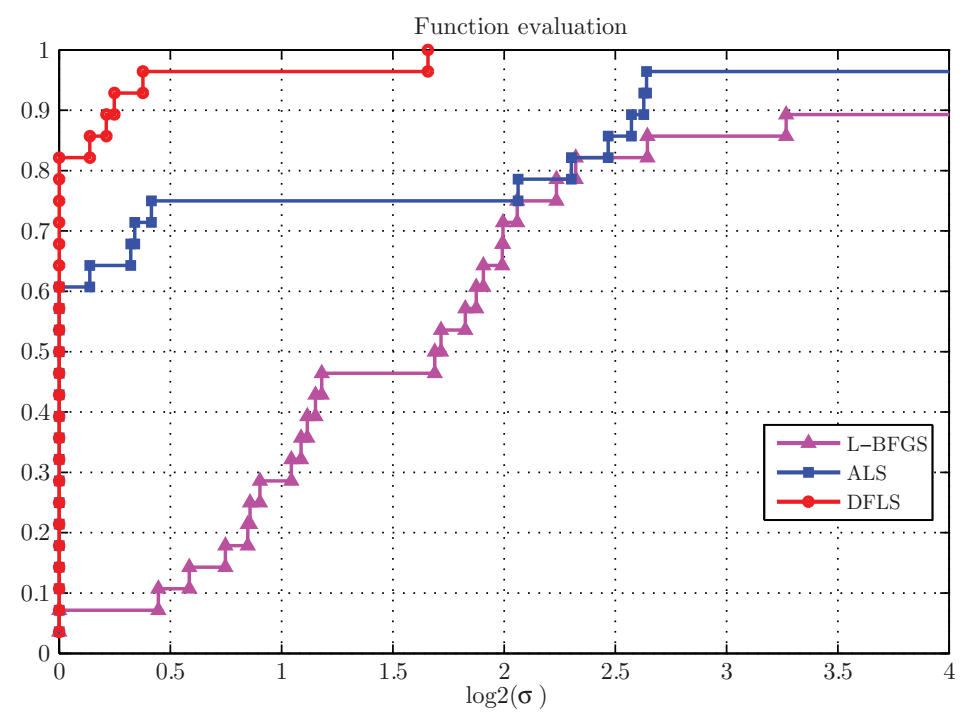

FiguRE 2. The performance profiles on the number of function evaluations for small-scale CUTE problems.

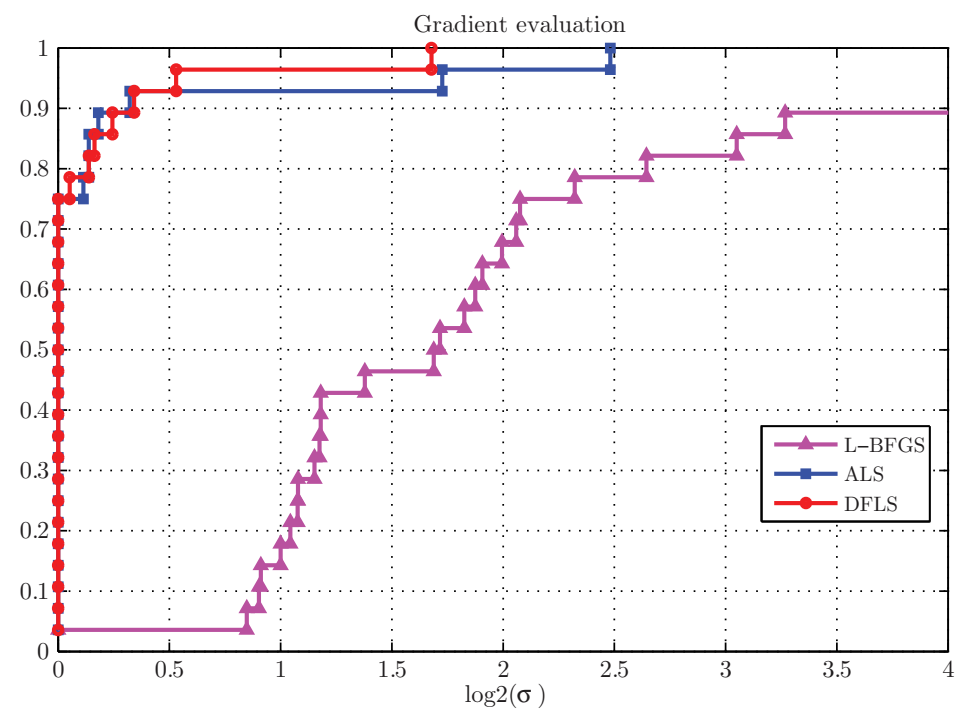

FiguRE 3. The performance profiles on the number of gradient evaluations for small-scale CUTE problems.

For these small-scale problems, the performance profiles of Algorithms DFLS, ALS and LBFGS on the numbers of function and gradient evaluations and CPU times are illustrated in Figures 2, 3 and 4, respectively. From the viewpoint of function and gradient evaluations, DFLS and ALS which exploit negative curvature directions from exact Hessians are more efficient than the first-order LBFGS algorithm. However, bath DFLS and ALS are slower than LBFGS as shown in 


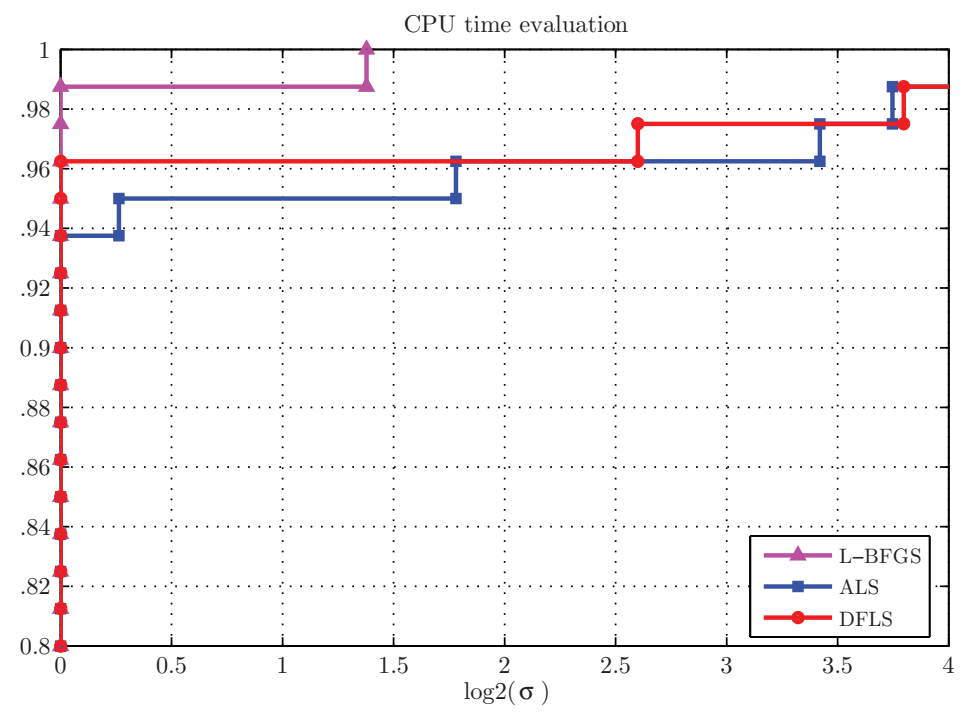

FiguRE 4 . The performance profiles on the CPU times for smallscale CUTE problems.

Figure 4. since the computation of the gradient-related direction and the negative curvature direction is more expensive than computing the search direction for LBFGS. We remark that LBFGS is even faster for large-scale problems. This is illustrated in Figure 7

Next, we focus on comparisons on Algorithms DFLS and ALS. Although more gradient evaluations are performed in dwindling filter steps, the performance of DFLS on the number of gradient evaluations is almost the same as ALS. Taking advantage of dwindling filter steps which provide more freedom to accept trial points, DFLS promotes the sequence of iterates to converge fast and saves many function evaluations.

For large-scale problems, we select twenty-four unconstrained problems from CUTE collection and list them in Table 2, To examine the robustness and efficiencies of Algorithms DFLS, ALS and LBFGS, we set the initial point of each problem as $10 \times x_{0}$, where $x_{0}$ is the default initial point. And these three algorithms return the same optimal function value. So we infer that the same critical point has been obtained for different algorithms. The performance profiles on the number of function and gradient evaluations and CPU times for large-scale problems are shown in Figures 5, 6 and 7. We find that DFLS saves more function and gradient evaluations than ALS. In particular, there are six problems in which DFLS outperforms ALS, while DFLS fails to do so only in one problem. So, we say that the DFLS algorithm is the best solver in these three algorithms.

When examining the remarkably successful example for the dwindling filter, we plot function values versus iterations on the EXTROSNB problem in Figure 8. DFLS solves this problem with $24 \%$ dwindling filter steps and does not clear out the filter. From Figure 8, we note that the sequence of function values does not decrease monotonously as iterations progress. Thanks to the nonmonotone, the EXTROSNB problem is rapidly solved when the dwindling filter is employed. 


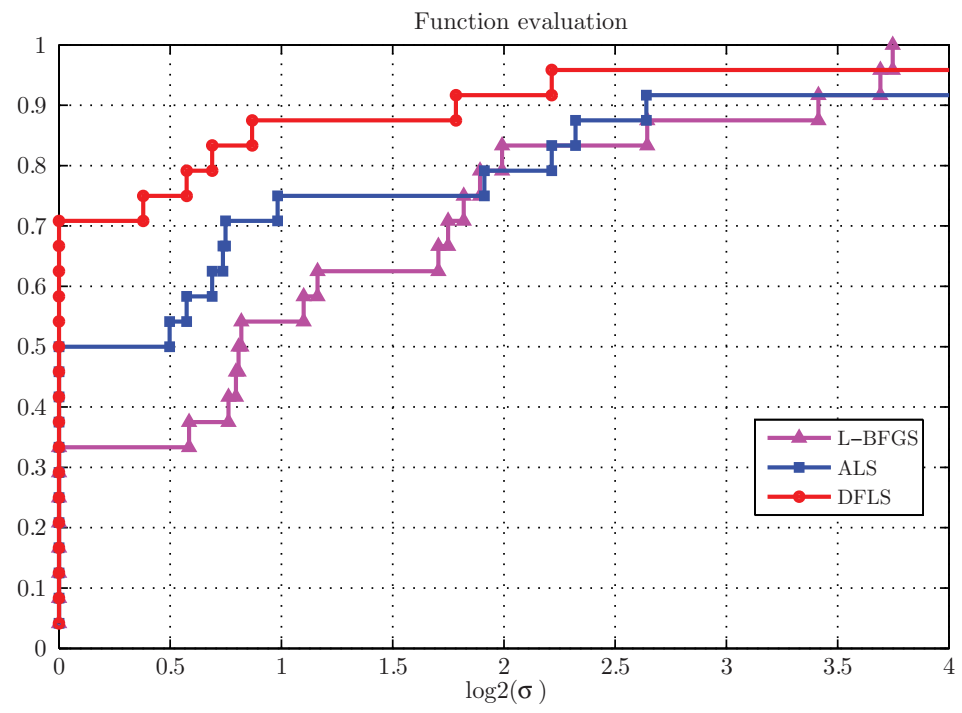

FiguRE 5. The performance profiles on the number of function evaluations for large-scale CUTE problems.

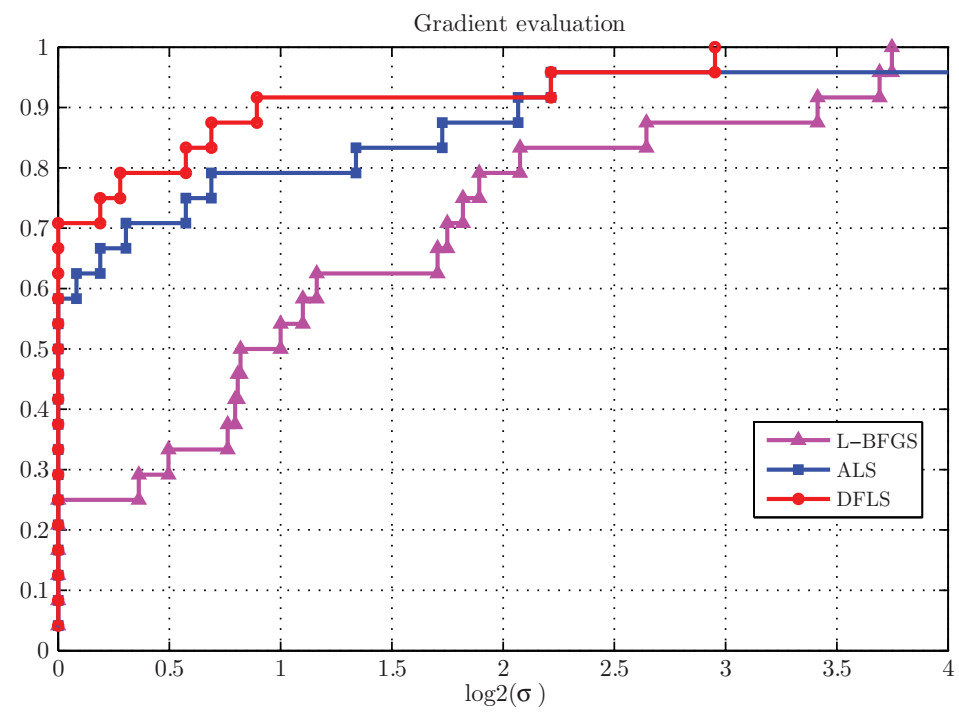

FiguRE 6. The performance profiles on the number of gradient evaluations for large-scale CUTE problems.

Finally, we list detailed numerical results for 80 small CUTE problems in Tables 3 and 4, and the numerical results for 24 large CUTE problems in Table 5 . These numerical results show that the algorithm DFLS is significantly efficient. 


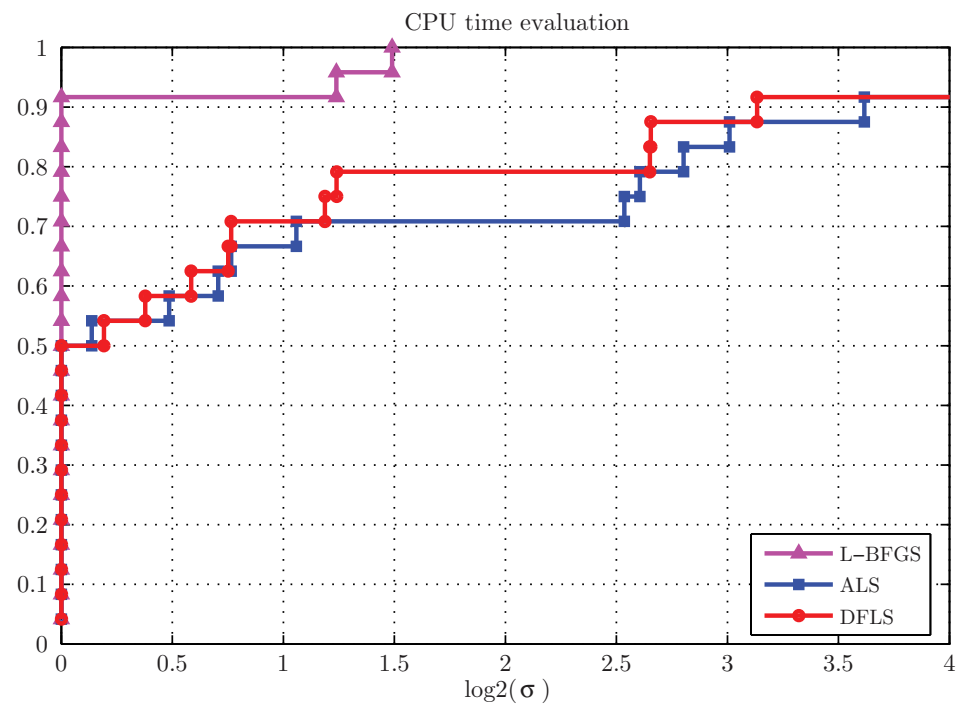

Figure 7 . The performance profiles on the CPU times for largescale CUTE problems.

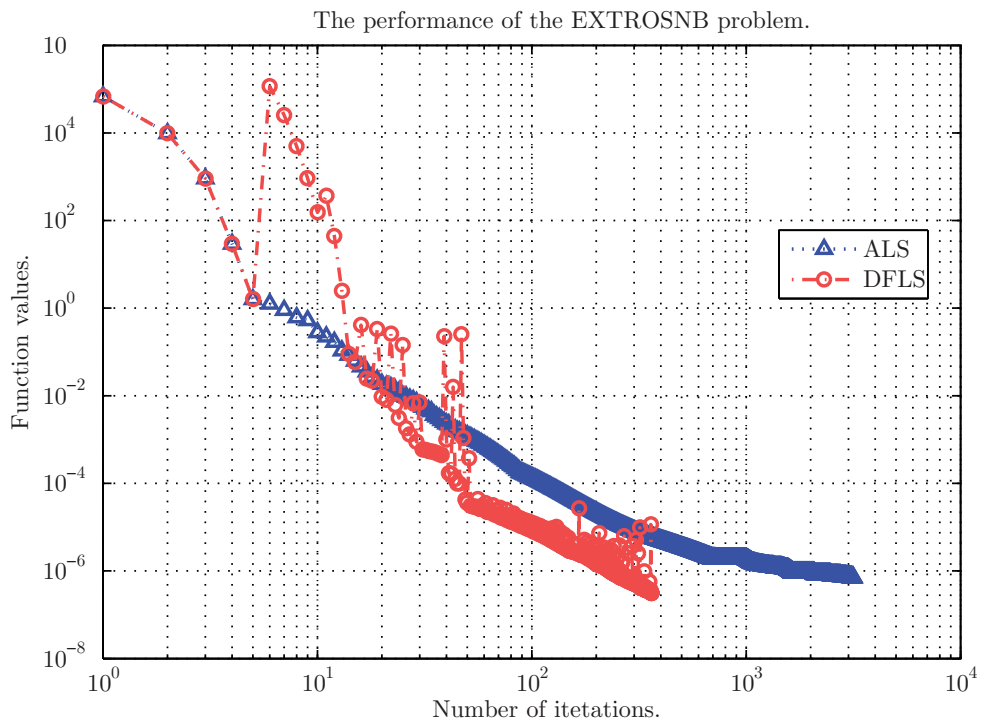

FiguRE 8. Function values versus iterations on the EXTROSNB problem. 
TABLE 3. Numerical results for the set of small CUTE problems.

\begin{tabular}{|c|c|c|c|c|c|c|c|c|c|c|c|c|}
\hline \multirow[b]{2}{*}{ No } & \multirow[b]{2}{*}{ Problem } & \multirow[b]{2}{*}{$\mathrm{n}$} & \multirow[b]{2}{*}{ Objective } & \multicolumn{3}{|c|}{ DFLS } & \multicolumn{3}{|c|}{ ALS } & \multicolumn{3}{|c|}{ L-BFGS } \\
\hline & & & & nf & ng & $\mathrm{cpu}$ & $\mathrm{nf}$ & ng & $\mathrm{cpu}$ & $\mathrm{nf}$ & ng & $\mathrm{cpu}$ \\
\hline 1 & AKIVA & 2 & $6.166 \mathrm{e}+00$ & 145 & 145 & 0.0 & 145 & 135 & 0.0 & 23 & 23 & 0.0 \\
\hline 2 & ALLINITU & 4 & $5.744 \mathrm{e}+00$ & 23 & 16 & 0.0 & 17 & 11 & 0.0 & 14 & 14 & 0.0 \\
\hline 3 & ARWHEAD & 100 & $0.000 \mathrm{e}+00$ & 7 & 7 & 0.0 & 72 & 6 & 0.0 & 12 & 12 & 0.0 \\
\hline 4 & BARD & 3 & $8.215 \mathrm{e}-03$ & 16 & 16 & 0.0 & 15 & 14 & 0.0 & 24 & 24 & 0.0 \\
\hline 5 & BDQRTIC & 100 & $3.788 \mathrm{e}+02$ & 14 & 14 & 0.0 & 14 & 14 & 0.0 & 111 & 111 & 0.0 \\
\hline 6 & BEALE & 2 & $1.774 \mathrm{e}-16$ & 17 & 11 & 0.0 & 15 & 10 & 0.0 & 16 & 16 & 0.0 \\
\hline 7 & BOX & 100 & $-1.124 \mathrm{e}+01$ & 7 & 6 & 0.0 & 7 & 6 & 0.0 & 13 & 13 & 0.0 \\
\hline 8 & BOX3 & 3 & $1.198 \mathrm{e}-12$ & 9 & 9 & 0.0 & 9 & 9 & 0.0 & 24 & 24 & 0.0 \\
\hline 9 & BRKMCC & 2 & $1.690 \mathrm{e}-01$ & 5 & 5 & 0.0 & 5 & 5 & 0.0 & 8 & 8 & 0.0 \\
\hline 10 & BROWNAL & 200 & $4.910 \mathrm{e}-10$ & 5 & 5 & 0.0 & 5 & 5 & 0.0 & 14 & 14 & 0.0 \\
\hline 11 & BROWNBS & 2 & $8.911 \mathrm{e}-14$ & 41 & 20 & 0.0 & 206 & 52 & 0.0 & 26 & 26 & 0.0 \\
\hline 12 & BROWNDEN & 4 & $8.582 \mathrm{e}+04$ & 12 & 12 & 0.0 & 15 & 13 & 0.0 & 44 & 44 & 0.0 \\
\hline 13 & BRYBND & 100 & $2.432 \mathrm{e}-14$ & 20 & 16 & 0.0 & 18 & 16 & 0.0 & 129 & 129 & 0.0 \\
\hline 14 & CHNROSNB & 50 & $1.952 \mathrm{e}-13$ & 66 & 53 & 0.0 & 158 & 86 & 0.0 & 298 & 298 & 0.0 \\
\hline 15 & CLIFF & 2 & $1.998 \mathrm{e}-01$ & 29 & 29 & 0.0 & 29 & 29 & 0.0 & 42 & 42 & 0.0 \\
\hline 16 & COSINE & 100 & $-9.900 e+01$ & 21 & 9 & 0.0 & 16 & 7 & 0.0 & 38 & 38 & 0.0 \\
\hline 17 & CUBE & 2 & $8.920 \mathrm{e}-13$ & 80 & 45 & 0.0 & 68 & 45 & 0.0 & 50 & 50 & 0.0 \\
\hline 18 & CURLY10 & 100 & $-1.003 e+04$ & 87 & 47 & 0.0 & 50 & 42 & 0.0 & 1029 & 1029 & 0.0 \\
\hline 19 & CURLY20 & 100 & $-1.003 e+04$ & 65 & 31 & 0.0 & 122 & 32 & 0.0 & 1132 & 1132 & 0.0 \\
\hline 20 & CURLY30 & 100 & $-1.003 e+04$ & 54 & 28 & 0.0 & 88 & 24 & 0.0 & 1294 & 1294 & 0.1 \\
\hline 21 & DENSCHNA & 2 & $2.739 \mathrm{e}-14$ & 7 & 7 & 0.0 & 7 & 7 & 0.0 & 11 & 11 & 0.0 \\
\hline 22 & DENSCHNB & 2 & $1.705 \mathrm{e}-14$ & 9 & 8 & 0.0 & 9 & 8 & 0.0 & 9 & 9 & 0.0 \\
\hline 23 & DENSCHNC & 2 & $1.757 \mathrm{e}-18$ & 13 & 13 & 0.0 & 13 & 13 & 0.0 & 18 & 18 & 0.0 \\
\hline 24 & DENSCHND & 3 & $1.254 \mathrm{e}-08$ & 4389 & 4205 & 0.0 & 4247 & 4191 & 0.0 & 65 & 65 & 0.0 \\
\hline 25 & DENSCHNE & 3 & $2.245 \mathrm{e}-15$ & 21 & 18 & 0.0 & 15 & 11 & 0.0 & 43 & 43 & 0.0 \\
\hline 26 & DENSCHNF & 2 & $2.270 \mathrm{e}-17$ & 7 & 7 & 0.0 & 7 & 7 & 0.0 & 10 & 10 & 0.0 \\
\hline 27 & DIXON3DQ & 100 & $1.010 \mathrm{e}-09$ & 95 & 95 & 0.0 & 95 & 95 & 0.0 & 661 & 661 & 0.0 \\
\hline 28 & DQDRTIC & 100 & $3.365 \mathrm{e}-15$ & 7 & 7 & 0.0 & 7 & 7 & 0.0 & 22 & 22 & 0.0 \\
\hline 29 & ENGVAL1 & 100 & $1.091 \mathrm{e}+02$ & 11 & 11 & 0.0 & 11 & 11 & 0.0 & 20 & 20 & 0.0 \\
\hline 30 & ENGVAL2 & 3 & $1.219 \mathrm{e}-14$ & 28 & 20 & 0.0 & 22 & 20 & 0.0 & 37 & 37 & 0.0 \\
\hline 31 & ERRINROS & 50 & $3.990 \mathrm{e}+01$ & 92 & 81 & 0.0 & 167 & 111 & 0.0 & 196 & 196 & 0.0 \\
\hline 32 & EXPFIT & 2 & $2.405 \mathrm{e}-01$ & 16 & 11 & 0.0 & 15 & 11 & 0.0 & 17 & 17 & 0.0 \\
\hline 33 & EXTROSNB & 100 & $3.068 \mathrm{e}-07$ & 871 & 794 & 0.1 & 85176 & 3687 & 1.1 & 13914 & 13914 & 0.3 \\
\hline 34 & FLETCBV2 & 100 & $-5.140 \mathrm{e}-01$ & 17 & 17 & 0.0 & 17 & 17 & 0.0 & 216 & 216 & 0.0 \\
\hline 35 & FLETCHCR & 100 & $3.781 \mathrm{e}-14$ & 143 & 141 & 0.0 & 295 & 197 & 0.0 & 605 & 605 & 0.0 \\
\hline 36 & FREUROTH & 100 & $1.196 \mathrm{e}+04$ & 49 & 38 & 0.0 & 127 & 31 & 0.0 & 50 & 50 & 0.0 \\
\hline 37 & GENROSE & 500 & $1.000 \mathrm{e}+00$ & 1317 & 530 & 1.0 & 599 & 336 & 0.6 & 1268 & 1268 & 0.2 \\
\hline 38 & GULF & 3 & $1.508 \mathrm{e}-09$ & 312 & 281 & 0.1 & 103 & 58 & 0.0 & 68 & 68 & 0.0 \\
\hline 39 & HAIRY & 2 & $2.000 \mathrm{e}+01$ & 5691 & 2330 & 0.0 & 3331 & 1487 & 0.0 & 92 & 92 & 0.0 \\
\hline 40 & HATFLDD & 3 & $6.631 \mathrm{e}-08$ & 31 & 27 & 0.0 & 30 & 28 & 0.0 & 24 & 24 & 0.0 \\
\hline
\end{tabular}


TABLE 4. Numerical results for the set of small CUTE problems (continued).

\begin{tabular}{|c|c|c|c|c|c|c|c|c|c|c|c|c|}
\hline \multirow[b]{2}{*}{ No } & \multirow[b]{2}{*}{ Problem } & \multirow[b]{2}{*}{$\mathrm{n}$} & \multirow[b]{2}{*}{ Objective } & \multicolumn{3}{|c|}{ DFLS } & \multicolumn{3}{|c|}{ ALS } & \multicolumn{3}{|c|}{ L-BFGS } \\
\hline & & & & $\mathrm{nf}$ & $\mathrm{ng}$ & cpu & $\mathrm{nf}$ & $\mathrm{ng}$ & cpu & $\mathrm{nf}$ & ng & $\mathrm{cpu}$ \\
\hline 41 & HATFLDE & 3 & $5.120 \mathrm{e}-07$ & 33 & 29 & 0.0 & 34 & 29 & 0.0 & 43 & 43 & 0.0 \\
\hline 42 & HATFLDFL & 3 & $6.226 \mathrm{e}-05$ & 89 & 39 & 0.0 & 141 & 39 & 0.0 & 818 & 818 & 0.0 \\
\hline 43 & HELIX & 3 & $4.911 \mathrm{e}-17$ & 28 & 19 & 0.0 & 28 & 20 & 0.0 & 33 & 33 & 0.0 \\
\hline 44 & HILBERTA & 2 & $9.208 \mathrm{e}-19$ & 3 & 3 & 0.0 & 3 & 3 & 0.0 & 7 & 7 & 0.0 \\
\hline 45 & HILBERTB & 10 & $2.292 \mathrm{e}-14$ & 6 & 6 & 0.0 & 6 & 6 & 0.0 & 7 & 7 & 0.0 \\
\hline 46 & HIMMELBB & 2 & $6.987 \mathrm{e}-14$ & 50 & 19 & 0.0 & 55 & 38 & 0.0 & 21 & 21 & 0.0 \\
\hline 47 & HIMMELBF & 4 & $3.186 e+02$ & 1997 & 1400 & 0.0 & 1606 & 1198 & 0.0 & 53 & 53 & 0.0 \\
\hline 48 & HIMMELBG & 2 & $7.288 \mathrm{e}-14$ & 99 & 43 & 0.0 & 389 & 190 & 0.0 & 13 & 13 & 0.0 \\
\hline 49 & HUMPS & 2 & $1.339 \mathrm{e}-12$ & 3217 & 1321 & 0.0 & 16961 & 8251 & 0.0 & 343 & 343 & 0.0 \\
\hline 50 & KOWOSB & 4 & $3.078 \mathrm{e}-04$ & 28 & 23 & 0.0 & 21 & 20 & 0.0 & 47 & 47 & 0.0 \\
\hline 51 & LIARWHD & 100 & $2.769 \mathrm{e}-14$ & 13 & 13 & 0.0 & 13 & 13 & 0.0 & 20 & 20 & 0.0 \\
\hline 52 & MARATOSB & 2 & $-1.000 e+00$ & 3631 & 2866 & 0.0 & 2666 & 1094 & 0.0 & 1528 & 1528 & 0.0 \\
\hline 53 & MEXHAT & 2 & $-4.001 e-02$ & 465 & 136 & 0.0 & 207 & 33 & 0.0 & 54 & 54 & 0.0 \\
\hline 54 & MOREBV & 100 & $4.507 \mathrm{e}-08$ & 6361 & 6361 & 2.9 & 6361 & 6361 & 2.8 & 9190 & 9190 & 0.2 \\
\hline 55 & NONDIA & 100 & $3.795 \mathrm{e}-21$ & 11 & 11 & 0.0 & 11 & 11 & 0.0 & 18 & 18 & 0.0 \\
\hline 56 & NONDQUAR & 100 & $4.680 \mathrm{e}-07$ & 152 & 148 & 0.0 & 5534 & 296 & 0.1 & 2183 & 2183 & 0.0 \\
\hline 57 & OSBORNEA & 5 & $5.465 \mathrm{e}-05$ & 62 & 41 & 0.0 & 176 & 133 & 0.0 & 194 & 194 & 0.0 \\
\hline 58 & OSBORNEB & 11 & $4.014 \mathrm{e}-02$ & 58 & 37 & 0.0 & 32 & 24 & 0.0 & 254 & 254 & 0.0 \\
\hline 59 & PALMER5C & 6 & $2.128 \mathrm{e}+00$ & 7 & 7 & 0.0 & 7 & 7 & 0.0 & 16 & 16 & 0.0 \\
\hline 60 & PENALTY1 & 100 & $9.025 \mathrm{e}-04$ & 25 & 25 & 0.0 & 50 & 40 & 0.0 & 70 & 70 & 0.0 \\
\hline 61 & PENALTY2 & 200 & $4.712 \mathrm{e}+13$ & 31 & 31 & 0.0 & 141 & 35 & 0.0 & 138 & 138 & 0.0 \\
\hline 62 & PENALTY3 & 200 & $9.987 \mathrm{e}-04$ & 3447 & 337 & 17.9 & 8120 & 427 & 28.7 & 96 & 96 & 0.7 \\
\hline 63 & POWELLSG & 100 & $8.281 \mathrm{e}-09$ & 18 & 18 & 0.0 & 18 & 18 & 0.0 & 68 & 68 & 0.0 \\
\hline 64 & POWER & 100 & $4.846 \mathrm{e}-10$ & 24 & 24 & 0.0 & 24 & 24 & 0.0 & 53 & 53 & 0.0 \\
\hline 65 & ROSENBR & 2 & $4.318 \mathrm{e}-14$ & 31 & 27 & 0.0 & 36 & 27 & 0.0 & 48 & 48 & 0.0 \\
\hline 66 & S308 & 2 & $7.732 \mathrm{e}-01$ & 12 & 12 & 0.0 & 12 & 12 & 0.0 & 14 & 14 & 0.0 \\
\hline 67 & SCHMVETT & 100 & $-2.940 e+02$ & 22 & 22 & 0.0 & 22 & 22 & 0.0 & 49 & 49 & 0.0 \\
\hline 68 & SINEVAL & 2 & $1.611 \mathrm{e}-16$ & 115 & 80 & 0.0 & 120 & 74 & 0.0 & 97 & 97 & 0.0 \\
\hline 69 & SISSER & 2 & $4.487 \mathrm{e}-10$ & 15 & 15 & 0.0 & 15 & 15 & 0.0 & 15 & 15 & 0.0 \\
\hline 70 & SPARSINE & 100 & $2.267 \mathrm{e}-13$ & 119 & 78 & 0.1 & 17 & 17 & 0.0 & 407 & 407 & 0.0 \\
\hline 71 & SPARSQUR & 100 & $1.863 \mathrm{e}-09$ & 18 & 18 & 0.0 & 18 & 18 & 0.0 & 26 & 26 & 0.0 \\
\hline 72 & TOINTGOR & 50 & $1.374 \mathrm{e}+03$ & 9 & 9 & 0.0 & 9 & 9 & 0.0 & 133 & 133 & 0.0 \\
\hline 73 & TOINTGSS & 100 & $1.010 \mathrm{e}+01$ & 22 & 22 & 0.0 & 22 & 22 & 0.0 & 33 & 33 & 0.0 \\
\hline 74 & TOINTPSP & 50 & $2.256 \mathrm{e}+02$ & 626 & 571 & 0.0 & 22 & 16 & 0.0 & 126 & 126 & 0.0 \\
\hline 75 & TOINTQOR & 50 & $1.175 \mathrm{e}+03$ & 8 & 8 & 0.0 & 8 & 8 & 0.0 & 43 & 43 & 0.0 \\
\hline 76 & TQUARTIC & 100 & $9.349 \mathrm{e}-12$ & 12 & 10 & 0.0 & 16 & 12 & 0.0 & 23 & 23 & 0.0 \\
\hline 77 & TRIDIA & 100 & $4.760 \mathrm{e}-14$ & 13 & 13 & 0.0 & 13 & 13 & 0.0 & 238 & 238 & 0.0 \\
\hline 78 & VAREIGVL & 50 & $1.757 \mathrm{e}-15$ & 7 & 7 & 0.0 & 7 & 7 & 0.0 & 29 & 29 & 0.0 \\
\hline 79 & WATSON & 12 & $3.151 \mathrm{e}-07$ & 53 & 53 & 0.0 & 27 & 15 & 0.0 & 2294 & 2294 & 0.0 \\
\hline 80 & YFITU & 3 & $2.080 \mathrm{e}-12$ & 150 & 125 & 0.0 & 123 & 83 & 0.0 & 98 & 98 & 0.0 \\
\hline
\end{tabular}

TABLE 5. Numerical results for the set of large CUTE problems with $10 \times x_{0}$.

\begin{tabular}{|c|c|c|c|c|c|c|c|c|c|c|c|c|}
\hline \multirow[b]{2}{*}{ No } & \multirow[b]{2}{*}{ Problem } & \multirow[b]{2}{*}{$\mathrm{n}$} & \multirow[b]{2}{*}{ Objective } & \multicolumn{3}{|c|}{ DFLS } & \multicolumn{3}{|c|}{$\overline{\mathrm{ALS}}$} & \multicolumn{3}{|c|}{ L-BFGS } \\
\hline & & & & $\mathrm{nf}$ & $\mathrm{ng}$ & $\mathrm{cpu}$ & $\mathrm{nf}$ & ng & cpu & $\mathrm{nf}$ & $\mathrm{ng}$ & $\mathrm{cpu}$ \\
\hline 1 & ARWHEAD & 5000 & $0.000 \mathrm{e}+00$ & 14 & 14 & 0.1 & 14 & $\overline{14}$ & 0.1 & 30 & 30 & 0.1 \\
\hline 2 & BDQRTIC & 5000 & $2.001 \mathrm{e}+04$ & 21 & 21 & 0.2 & 21 & 21 & 0.2 & 282 & 282 & 0.6 \\
\hline 3 & $\mathrm{BOX}$ & 10000 & $-1.865 e+03$ & 8 & 6 & 0.1 & 8 & 6 & 0.1 & 12 & 12 & 0.1 \\
\hline 4 & BRYBND & 5000 & $7.264 \mathrm{e}-15$ & 108 & 108 & 1.6 & 108 & 108 & 1.5 & 67 & 67 & 0.2 \\
\hline 5 & COSINE & 10000 & $-9.999 e+03$ & 31 & 13 & 0.1 & 15 & 7 & 0.1 & 9 & 9 & 0.0 \\
\hline 6 & DQDRTIC & 5000 & $1.074 \mathrm{e}-13$ & 7 & 7 & 0.0 & 7 & 7 & 0.0 & 26 & 26 & 0.0 \\
\hline 7 & EDENSCH & 2000 & $1.200 \mathrm{e}+04$ & 21 & 21 & 0.1 & 21 & 21 & 0.0 & 47 & 47 & 0.0 \\
\hline 8 & $\mathrm{EG} 2$ & 1000 & $-9.989 e+02$ & 4 & 4 & 0.0 & 4 & 4 & 0.0 & 25 & 25 & 0.0 \\
\hline 9 & ENGVAL1 & 5000 & $5.549 \mathrm{e}+03$ & 17 & 17 & 0.1 & 85 & 18 & 0.1 & 30 & 30 & 0.1 \\
\hline 10 & EXTROSNB & 1000 & $5.826 \mathrm{e}-07$ & 1028 & 1028 & 1.0 & 112617 & 4308 & 12.3 & 13290 & 13290 & 2.4 \\
\hline 11 & FLETCHCR & 1000 & $1.372 \mathrm{e}-12$ & 1426 & 1345 & 3.3 & 8889 & 4452 & 10.1 & 5672 & 5672 & 1.4 \\
\hline 12 & LIARWHD & 5000 & $1.027 \mathrm{e}-12$ & 23 & 23 & 0.1 & 23 & 23 & 0.1 & 39 & 39 & 0.1 \\
\hline 13 & MOREBV & 5000 & $3.032 \mathrm{e}-08$ & 67 & 67 & 1.7 & 67 & 67 & 1.7 & 45 & 45 & 0.1 \\
\hline 14 & NONDIA & 5000 & $3.352 \mathrm{e}-12$ & 34 & 34 & 0.1 & 48 & 42 & 0.1 & 59 & 59 & 0.1 \\
\hline 15 & PENALTY1 & 1000 & $9.686 \mathrm{e}-03$ & 157 & 74 & 0.0 & 170 & 61 & 0.0 & 86 & 86 & 0.0 \\
\hline 16 & POWELLSG & 5000 & $8.722 \mathrm{e}-07$ & 23 & 23 & 0.1 & 23 & 23 & 0.1 & 75 & 75 & 0.1 \\
\hline 17 & POWER & 10000 & $5.339 \mathrm{e}-10$ & 58 & 58 & 2.0 & 58 & 58 & 1.9 & 618 & 618 & 1.2 \\
\hline 18 & SCHMVETT & 5000 & $-1.499 \mathrm{e}+04$ & 1126 & 178 & 9.1 & 22125 & 6846 & 20.7 & 23 & 23 & 0.1 \\
\hline 19 & SPARSQUR & 10000 & $1.436 \mathrm{e}-08$ & 28 & 28 & 0.8 & 28 & 28 & 0.8 & 49 & 49 & 0.4 \\
\hline 20 & SROSENBR & 5000 & $5.481 \mathrm{e}-13$ & 17 & 17 & 0.0 & 64 & 43 & 0.1 & 60 & 60 & 0.1 \\
\hline 21 & TOINTGSS & 5000 & $1.000 \mathrm{e}+01$ & 65 & 65 & 0.6 & 65 & 65 & 0.6 & 14 & 14 & 0.0 \\
\hline 22 & TQUARTIC & 5000 & $0.000 \mathrm{e}+00$ & 1 & 1 & 0.0 & 1 & 1 & 0.0 & 1 & 1 & 0.0 \\
\hline 23 & TRIDIA & 5000 & $2.263 \mathrm{e}-13$ & 596 & 596 & 11.4 & 596 & 596 & 11.1 & 2004 & 2004 & 1.8 \\
\hline 24 & WOODS & 4000 & $1.255 \mathrm{e}-12$ & 65 & 57 & 0.2 & 84 & 57 & 0.2 & 50 & 50 & 0.1 \\
\hline
\end{tabular}




\section{Conclusion}

In this paper, a dwindling multidimensional filter technique is presented in a line search algorithm framework for solving large-scale unconstrained optimization problems. Under some mild assumptions, we prove that the new algorithm converges to the first-order and second-order critical points. Preliminary numerical experiments and numerical comparison with ALS and L-BFGS indicate that the new algorithm is robust and efficient.

\section{ACKNOWLEDGEMENTS}

The authors thank Professor Ph. L. Toint for his helpful advice on using CUTE problems. The authors also appreciate sincerely two referees for their valuable comments and suggestions which improved our manuscript essentially.

\section{REFERENCES}

[1] I. Bongartz, A. R. Conn, Nicholas I. M. Gould, and Philippe L. Toint, Cute: constrained and unconstrained testing environment, ACM Transactions on Mathematical Software 21 (1995), no. 1, 123-160.

[2] Yan Nan Chen and Wen Yu Sun, A new nonmonotone optimization method with trust region and second-order line search (Chinese, with English summary), Numer. Math. J. Chinese Univ. 32 (2010), no. 4, 369-386. MR2790772 (2011m:90154)

[3] Elizabeth D. Dolan and Jorge J. Moré, Benchmarking optimization software with performance profiles, Math. Program. 91 (2002), no. 2, Ser. A, 201-213, DOI 10.1007/s101070100263. MR.1875515 (2002j:90001)

[4] G. Fasano, Planar conjugate gradient algorithm for large-scale unconstrained optimization. I. Theory, J. Optim. Theory Appl. 125 (2005), no. 3, 523-541, DOI 10.1007/s10957-005-2087-1. MR.2148660(2006a:90163)

[5] G. Fasano, Planar conjugate gradient algorithm for large-scale unconstrained optimization. II. Application, J. Optim. Theory Appl. 125 (2005), no. 3, 543-558, DOI 10.1007/s10957005-2088-0. MR2148661(2006a:90164)

[6] Giovanni Fasano and Massimo Roma, Iterative computation of negative curvature directions in large scale optimization, Comput. Optim. Appl. 38 (2007), no. 1, 81-104, DOI 10.1007/s10589-007-9034-z. MR2332402(2008i:90084)

[7] M. C. Ferris, S. Lucidi, and M. Roma, Nonmonotone curvilinear line search methods for unconstrained optimization, Comput. Optim. Appl. 6 (1996), no. 2, 117-136, DOI 10.1007/BF00249642. MR1398263 (97d:90087)

[8] Roger Fletcher, Nicholas I. M. Gould, Sven Leyffer, Philippe L. Toint, and Andreas Wächter, Global convergence of a trust-region SQP-filter algorithm for general nonlinear programming, SIAM J. Optim. 13 (2002), no. 3, 635-659 (2003), DOI 10.1137/S1052623499357258. MR.1972208 (2004b:90105)

[9] Roger Fletcher and Sven Leyffer, Nonlinear programming without a penalty function, Math. Program. 91 (2002), no. 2, Ser. A, 239-269, DOI 10.1007/s101070100244. MR.1875517 (2002j:90074)

[10] Roger Fletcher, Sven Leyffer, and Philippe L. Toint, On the global convergence of a filter-SQP algorithm, SIAM J. Optim. 13 (2002), no. 1, 44-59 (electronic), DOI 10.1137/S105262340038081X. MR.1922433 (2003g:90121)

[11] Roger Fletcher, Sven Leyffer, and Philippe L. Toint, A brief history of filter methods, SIAG/OPT Views-and-News 18 (2007), no. 1, 2-12.

[12] Clóvis C. Gonzaga, Elizabeth Karas, and Márcia Vanti, A globally convergent filter method for nonlinear programming, SIAM J. Optim. 14 (2003), no. 3, 646-669 (electronic), DOI 10.1137/S1052623401399320. MR2085935(2005d:49049)

[13] Nicholas I. M. Gould, Sven Leyffer, and Philippe L. Toint, A multidimensional filter algorithm for nonlinear equations and nonlinear least-squares, SIAM J. Optim. 15 (2004), no. 1, 17-38, DOI 10.1137/S1052623403422637. MR2112974 (2005m:90117) 
[14] N. I. M. Gould, S. Lucidi, M. Roma, and Ph. L. Toint, Exploiting negative curvature directions in linesearch methods for unconstrained optimization, Optim. Methods Softw. 14 (2000), no. 1-2, 75-98, DOI 10.1080/10556780008805794. International Conference on Nonlinear Programming and Variational Inequalities (Hong Kong, 1998). MR.1809604 (2001m:90104)

[15] Nicholas I. M. Gould, Dominique Orban, and Philippe L. Toint, GALAHAD, a library of thread-safe Fortran 90 packages for large-scale nonlinear optimization, ACM Trans. Math. Software 29 (2003), no. 4, 353-372, DOI 10.1145/962437.962438. MR2077337 (2005b:90006)

[16] Nick I. M. Gould, Caroline Sainvitu, and Philippe L. Toint, A filter-trust-region method for unconstrained optimization, SIAM J. Optim. 16 (2005), no. 2, 341-357, DOI 10.1137/040603851. MR2197983 (2006i:90076)

[17] Nataša Krejić, Zorana Lužanin, and Irena Stojkovska, Gauss-Newton-based BFGS methods with filter for unconstrained minimization, Appl. Math. Comput. 211 (2009), no. 2, 354-362, DOI 10.1016/j.amc.2009.01.041. MR.2524164

[18] ChengJin Li and WenYu Sun, On filter-successive linearization methods for nonlinear semidefinite programming, Sci. China Ser. A 52 (2009), no. 11, 2341-2361, DOI 10.1007/s11425009-0168-6. MR2566650 (2010j:90104)

[19] Shujun Li and Zhenhai Liu, A new trust region filter algorithm, Appl. Math. Comput. 204 (2008), no. 1, 485-489, DOI 10.1016/j.amc.2008.07.007. MR.2458387

[20] Dong C. Liu and Jorge Nocedal, On the limited memory BFGS method for large scale optimization, Math. Programming 45 (1989), no. 3, (Ser. B), 503-528, DOI 10.1007/BF01589116. MR.1038245 (90m:90253)

[21] Jun Long and Sanyun Zeng, A new filter-Levenberg-Marquardt method with disturbance for solving nonlinear complementarity problems, Appl. Math. Comput. 216 (2010), no. 2, 677688, DOI 10.1016/j.amc.2010.01.098. MR2601536

[22] Stefano Lucidi, Francesco Rochetich, and Massimo Roma, Curvilinear stabilization techniques for truncated Newton methods in large scale unconstrained optimization, SIAM J. Optim. 8 (1998), no. 4, 916-939 (electronic), DOI 10.1137/S1052623495295250. MR.1641270 (2000d:90073)

[23] Garth P. McCormick, A modification of Armijo's step-size rule for negative curvature, Math. Programming 13 (1977), no. 1, 111-115. MR0461907 (57 \#1889)

[24] Wei Hua Miao and Wen Yu Sun, A filter trust-region method for unconstrained optimization (Chinese, with English summary), Numer. Math. J. Chinese Univ. 29 (2007), no. 1, 88-96. MR2337211(2009g:90147)

[25] Jorge J. Moré and Danny C. Sorensen, On the use of directions of negative curvature in a modified Newton method, Math. Programming 16 (1979), no. 1, 1-20, DOI 10.1007/BF01582091. MR517757 (80b:90119)

[26] Pu-yan Nie, Ming-yong Lai, Shu-jin Zhu, and Pei-ai Zhang, A line search filter approach for the system of nonlinear equations, Comput. Math. Appl. 55 (2008), no. 9, 2134-2141, DOI 10.1016/j.camwa.2007.08.037. MR2401120(2009b:90145)

[27] Jorge Nocedal, Updating quasi-Newton matrices with limited storage, Math. Comp. 35 (1980), no. 151, 773-782, DOI 10.2307/2006193. MR572855 (81g:65077)

[28] Jorge Nocedal and Stephen J. Wright, Numerical optimization, 2nd ed., Springer Series in Operations Research and Financial Engineering, Springer, New York, 2006. MR2244940 (2007a:90001)

[29] Ademir A. Ribeiro, Elizabeth W. Karas, and Clóvis C. Gonzaga, Global convergence of filter methods for nonlinear programming, SIAM J. Optim. 19 (2008), no. 3, 1231-1249, DOI 10.1137/060672285. MR2460740 (2010f:90164)

[30] Caroline Sainvitu and Philippe L. Toint, A filter-trust-region method for simple-bound constrained optimization, Optim. Methods Softw. 22 (2007), no. 5, 835-848, DOI 10.1080/10556780701322970. MR2354770 (2008g:90116)

[31] Wenyu Sun, Filter methods for optimization: motivation and development, invited talk, International Conference on Engineering Mathematics and Computational Mathematics, Hong Kong Polytechnical University, December 2009.

[32] Wenyu Sun and Ya xiang Yuan, Optimization Theory and Methods: Nonlinear Programming, Springer Optimization and Its Applications, vol. 1, Springer, New York, 2006.

[33] Wen-yu Sun and Qun-yan Zhou, An unconstrained optimization method using nonmonotone second order Goldstein's line search, Sci. China Ser. A 50 (2007), no. 10, 1389-1400, DOI 10.1007/s11425-007-0072-x. MR2390457(2009b:90147) 
[34] Andreas Wächter and Lorenz T. Biegler, Line search filter methods for nonlinear programming: local convergence, SIAM J. Optim. 16 (2005), no. 1, 32-48 (electronic), DOI 10.1137/S1052623403426544. MR2177768 (2006e:90121)

[35] Andreas Wächter and Lorenz T. Biegler, Line search filter methods for nonlinear programming: motivation and global convergence, SIAM J. Optim. 16 (2005), no. 1, 1-31 (electronic), DOI 10.1137/S1052623403426556. MR2177767 (2006e:90120)

[36] Zhujun Wang and Detong Zhu, A filter-line-search method for unconstrained optimization, J. Appl. Math. Comput. 34 (2010), no. 1-2, 329-342, DOI 10.1007/s12190-009-0324-8. MR2718789(2012a:90179)

[37] Zhenghao Yang, Wenyu Sun, and Chuangyin Dang, A filter-trust-region method for $L C^{1}$ unconstrained optimization and its global convergence, Anal. Theory Appl. 24 (2008), no. 1, 55-66, DOI 10.1007/s10496-008-0055-y. MR2422460 (2009g:90117)

[38] Yan Zhang, Wenyu Sun, and Liqun Qi, A nonmonotone filter Barzilai-Borwein method for optimization, Asia-Pac. J. Oper. Res. 27 (2010), no. 1, 55-69, DOI 10.1142/S0217595910002582. MR2646952(2011k:90188)

[39] Qun-yan Zhou and Wen-yu Sun, An adaptive nonmonotonic trust region method with curvilinear searches, J. Comput. Math. 24 (2006), no. 6, 761-770. MR2269958 (2007f:90108)

[40] Qun-yan Zhou and Wen-yu Sun, A nonmonotone second-order steplength method for unconstrained minimization, J. Comput. Math. 25 (2007), no. 1, 104-112. MR2292432 (2007j:90073)

School of Mathematical Sciences, Jiangsu Key Laboratory for NSLSCS, Nanjing Normal University, Nanjing 210046, China; and School of Mathematics and Statistics, Zhengzhou University, Zhengzhou 450001, China

E-mail address: ynchen@zzu.edu.cn

Corresponding author: School of Mathematical Sciences, Jiangsu Key Laboratory For NSLSCS, NANJing Normal University, NANJing 210046, China

E-mail address: wysun@njnu.edu.cn 